NASA Contractor Report 182061

ICASE Report No. 90-44

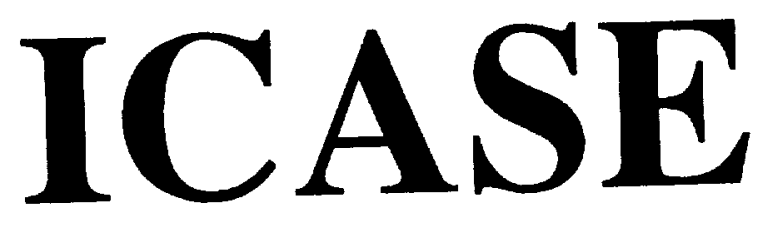

\title{
ON CENTRAL-DIFFERENCE AND UPWIND SCHEMES
}

\author{
R. C. Swanson \\ Eli Turkel
}

Contract No. NAS1-18605

June 1990

Institute for Computer Applications in Science and Engineering NASA Langley Research Center

Hampton, Virginia 23665-5225

Operated by the Universities Space Research Association

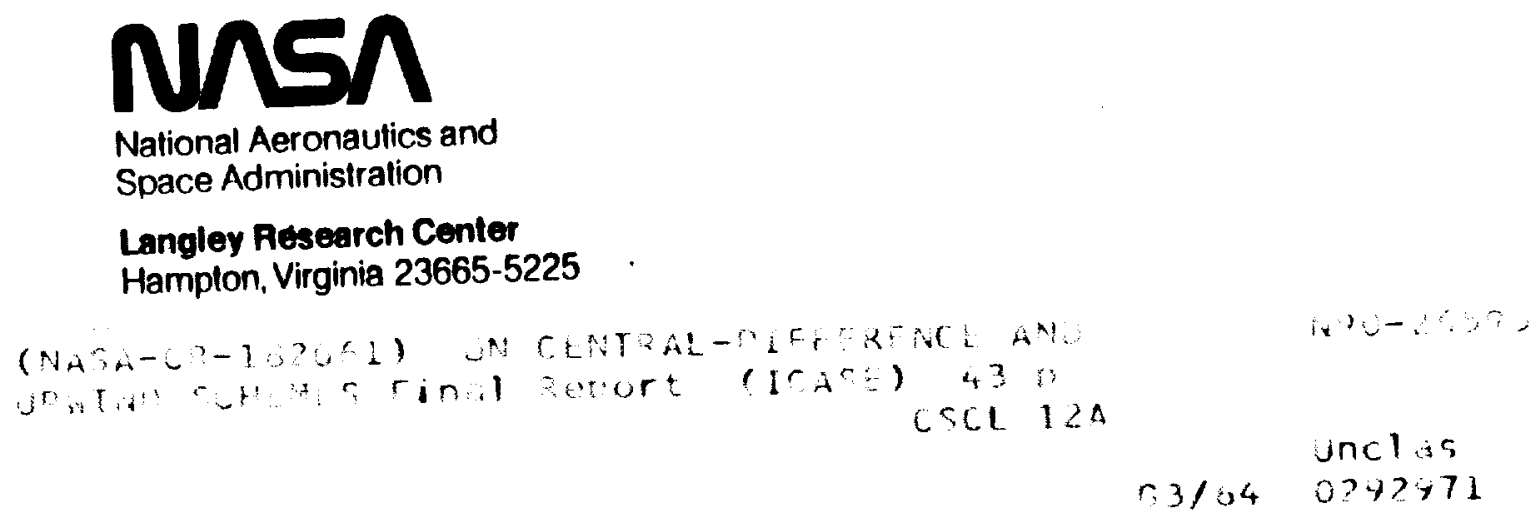




$-$




\title{
ON CENTRAL-DIFFERENCE AND UPWIND SCHEMES
}

\author{
R. C. Swanson \\ NASA Langley Research Center \\ Hampton, VA 23665 \\ Eli Turkel ${ }^{1}$ \\ Institute for Computer Applications in Science and Engineering \\ NASA Langley Research Center \\ Hampton, VA 23665 \\ and \\ Tel-Aviv University \\ Tel-Aviv, Israel
}

\begin{abstract}
A class of numerical dissipation models for central-difference schemes constructed with second- and fourth-difference terms is considered. The notion of matrix dissipation associated with upwind schemes is used to establish improved shock capturing capability for these models. In addition, conditions are given that guarantee that such dissipation models produce a TVD scheme. Appropriate switches for this type of model to ensure satisfaction of the TVD property are presented. Significant improvements in the accuracy of a centraldifference scheme are demonstrated by computing both inviscid and viscous transonic airfoil flows.
\end{abstract}

\footnotetext{
${ }^{1}$ Research was supported by the National Aeronautics and Space Administration under NASA Contract No. NAS1-18605 while the author was in residence at the Institute for Computer Applications in Science and Engineering (ICASE), NASA Langley Research Center, Hampton, VA 23665.
} 



\section{Introduction}

Central difference type schemes are currently being applied on a regular basis in the solution of the Euler and Navier-Stokes equations. A numerical dissipation model is included in these schemes, and it plays a crucial role in the determination of their success. The form of the dissipation model is quite often a blending of second-difference and fourthdifference dissipation terms. The second-difference terms are used to prevent oscillations at shock waves, while the fourth-difference terms are important for stability and convergence to a steady state. There is a constant to be specified for each contribution. However, using the model in conjunction with appropriate numerical procedures, these constants can usually be selected and maintained for a fairly wide class of fluid dynamics problems. This dissipation model allows shock waves to be captured with smearing over three to four mesh cells.

Even though these central-difference schemes have proven to be reasonably effective in many cases, there are still strong motivations for reducing the numerical dissipation being produced. For example, by appropriate reduction of the artificial dissipation, shock wave representation and boundary-layer definition (especially the wall shear stresses) can be improved on coarse meshes. Such improvements in accuracy are especially beneficial for complex three-dimensional flows, which can demand extensive computational effort. In addition, better estimates of the limit of infinitely fine mesh values of aerodynamic coefficients for flows with shocks can be obtained. Also, the standard model has difficulties in hypersonic flow. Finally, for some problems, the influence of numerical dissipation needs to be severely limited in certain smooth regions of a flow field (i.e., near the trailing edge of an airfoil), while still maintaining stability near discontinuities. This difficulty cannot generally be resolved by simply reducing the global constants in the dissipation model.

One can appeal to ideas from upwind schemes to improve the dissipation model, especially in the vicinity of shock waves. Upwind algorithms utilize concepts from characteristic theory in order to determine the direction of spatial differencing. They have been extended to systems of conservation laws using such approaches as the flux-vector splitting of van Leer [1] and the approximate Riemann solver of Roe [2]. A fundamental feature of these schemes is the use of a matrix evaluation of the dissipation either in the implied or direct sense. In so doing, the dissipative terms of each discrete equation are scaled by the appropriate eigenvalues of the flux Jacobian matrices of the Euler equations, rather than the same eigenvalue as in the dissipation model employed with central-difference 
schemes. Also, upwind schemes can be designed to have the total variation diminishing (TVD) property, which prevents the occurrence of spurious oscillations. The disadvantage of these schemes is that, in general, they increase the operational count for processing mesh points by about a factor of two over that required by central-difference schemes. One would certainly like to more closely imitate the highly desirable behavior of the upwind algorithms near flow discontinuities, and at the same time, retain the more efficient central-difference scheme over significant portions of a flow field. In addition, one would like to have the high degree of numerical efficiency that has been achieved by combining a central-difference scheme with a Runge-Kutta time marching algorithm, which includes residual smoothing and multigrid acceleration techniques.

The primary purpose of this paper is to construct a numerical dissipation model for a central-difference scheme that has both the properties of matrix dissipation and of TVD. As a starting point, we consider the elements of a widely used scalar dissipation model. Modifications of this model that facilitate accurate viscous flow computations are also examined. In the next section of the paper, the intimate connection between the formulation for an upwind scheme and a centered-difference scheme is presented, so as to establish a foundation for a matrix dissipation model. Then a theorem is proved that provides a simple sufficient condition to determine when a central-difference scheme with dissipation terms comprised of second and fourth differences is TVD. In the following section, appropriate flux limiter functions consistent with the central-difference dissipation model are discussed. A multistage time-stepping scheme used in applications is next briefly described. Finally, numerical results are shown to demonstrate the benefits of using the matrix dissipation model. Both inviscid and viscous transonic airfoil flows are computed.

\section{Scalar Dissipation Model}

The basic elements of the scalar dissipation model considered in this paper were first introduced by Jameson, Schmidt, and Turkel [3] in conjunction with Runge-Kutta explicit schemes. This dissipation model has been used by many investigators [4-6] to numerically solve the Euler equations for a wide range of fluid dynamic applications. The same type of dissipation model has been applied to alternating direction implicit (ADI) schemes [7] and LU factored implicit schemes [8]. Several modifications of the model have been investigated in [9] and [10] in order to improve it and make it suitable for obtaining accurate and efficient solutions of the Navier-Stokes equations. In this section, the basic model and important 
modifications are briefly reviewed.

Consider the Euler equations in the form

$$
W_{t}+f_{x}+g_{y}=0
$$

where $W$ is the four-component vector of conserved variables, and $f, g$ are the flux vectors. The independent variables are time $t$ and Cartesian coordinates $(x, y)$. If $(2.1)$ is transformed to arbitrary curvilinear coordinates $\xi=\xi(x, y)$ and $\eta=\eta(x, y)$, then we obtain

$$
\left(J^{-1} W\right)_{t}+F_{\xi}+G_{\eta}=0
$$

where $J^{-1}$ is the inverse transformation Jacobian, and

$$
F=f y_{\eta}-g x_{\eta}, \quad G=g x_{\xi}-f y_{\xi} .
$$

In a cell-centered, finite-volume method, $(2.2)$ is integrated over an elemental volume in the discretized computational domain, and $J^{-1}$ is identified as the volume of the cell. Equation (2.2) can also be written as

$$
J^{-1} W_{t}+A W_{\xi}+B W_{\eta}=0
$$

where $A$ and $B$ are the flux Jacobian matrices defined by $A=\partial F / \partial W$ and $B=\partial G / \partial W$.

To advance the scheme in time we use a multistage scheme. A typical step of a Runge-Kutta approximation to (2.2) is

$$
W^{(k)}=W^{(o)}-\alpha_{k} \frac{\Delta t}{J^{-1}}\left[D_{\xi} F^{(k-1)}+D_{\eta} G^{(k-1)}-A D\right]
$$

where $D_{\xi}$ and $D_{\eta}$ are spatial differencing operators, and $A D$ represents the artificial dissipation terms. The dissipation terms are a blending of second and fourth differences. That is,

$$
A D=\left(D_{\xi}^{2}+D_{\eta}^{2}-D_{\xi}^{4}-D_{\eta}^{4}\right) W
$$

where

$$
\begin{gathered}
D_{\xi}^{2} W=\nabla_{\xi}\left[\left(\lambda_{i+\frac{1}{2}, j} \epsilon_{i+\frac{1}{2}, j}^{(2)}\right) \Delta_{\xi}\right] W_{i, j}, \\
D_{\xi}^{4} W=\nabla_{\xi}\left[\left(\lambda_{i+\frac{1}{2}, j} \epsilon_{i+\frac{1}{2}, j}^{(4)}\right) \Delta_{\xi} \nabla_{\xi} \Delta_{\xi}\right] W_{i, j},
\end{gathered}
$$


and $\Delta_{\xi}, \nabla_{\xi}$ are the standard forward and backward difference operators respectively associated with the $\xi$ direction. The variable scaling factor $\lambda$ was originally chosen as

$$
\lambda_{i+\frac{1}{2}, j}=\frac{1}{2}\left[\left(\lambda_{\xi}\right)_{i, j}+\left(\lambda_{\xi}\right)_{i+1, j}+\left(\lambda_{\eta}\right)_{i, j}+\left(\lambda_{\eta}\right)_{i+1, j}\right],
$$

where $\lambda_{\xi}$ and $\lambda_{\eta}$ are proportional to the largest eigenvalues of the matrices $A$ and $B$. The scaled spectral radii $\lambda_{\xi}$ and $\lambda_{\eta}$ are given by

$$
\begin{aligned}
& \lambda_{\xi}=\left|u y_{\eta}-v x_{\eta}\right|+c \sqrt{x_{\eta}^{2}+y_{\eta}^{2}}, \\
& \lambda_{\eta}=\left|v x_{\xi}-u y_{\xi}\right|+c \sqrt{x_{\xi}^{2}+y_{\xi}^{2}},
\end{aligned}
$$

where $u$ and $v$ are the Cartesian velocity components, and $c$ is the speed of sound. The coefficients $\epsilon^{(2)}$ and $\epsilon^{(4)}$ are adapted to the flow and are defined as follows:

$$
\begin{gathered}
\epsilon_{i+\frac{1}{2}, j}^{(2)}=\kappa^{(2)} \max \left(\nu_{i-1, j}, \nu_{i, j}, \nu_{i+1, j}, \nu_{i+2, j}\right) \\
\nu_{i, j}=\left|\frac{p_{i+1, j}-2 p_{i, j}+p_{i-1, j}}{p_{i+1, j}+2 p_{i, j}+p_{i-1, j}}\right| \\
\epsilon_{i+\frac{1}{2}, j}^{(4)}=\max \left[0,\left(\kappa^{(4)}-\epsilon_{i+\frac{1}{2}, j}^{(2)}\right)\right]
\end{gathered}
$$

where $p$ is the pressure, and the quantities $\kappa^{(2)}$ and $\kappa^{(4)}$ are constants to be specified. The operators in (2.4) for the $\eta$ direction are defined in a similar manner.

The second-difference dissipation term is nonlinear. Its purpose is to introduce an entropy-like condition and to suppress oscillations in the neighborhood of shocks. This term is small in the smooth portion of the flow field. The fourth-difference dissipation term is basically linear and is included to damp high-frequency modes and allow the scheme to approach a steady state. Only this term affects the linear stability of the scheme. Near shocks it is reduced to zero.

The isotropic scaling factor of the original dissipation model as given in (2.7) is generally satisfactory for inviscid flow problems when typical inviscid flow meshes (i.e., cell aspect ratio $O(1))$ are used. The factor can produce too much numercial dissipation in the cases of meshes with high aspect ratio cells. This is also an important consideration for high Reynolds number viscous flows, where a mesh providing appropriate spatial resolution 
can have cell aspect ratios $O\left(10^{3}\right)$. In [9] and [10] this difficulty is remedied by replacing the factor of (2.7) with the anisotropic one

$$
\lambda_{i+\frac{1}{2}, j}=\frac{1}{2}\left[\left(\bar{\lambda}_{\xi}\right)_{i, j}+\left(\bar{\lambda}_{\xi}\right)_{i+1, j}\right]
$$

where

$$
\begin{array}{cl}
\left(\bar{\lambda}_{\xi}\right)_{i, j}=\phi_{i, j}(r) & \left(\lambda_{\xi}\right)_{i, j}, \\
\phi_{i, j}(r)=1+r_{i, j}^{\zeta} & 0<\zeta<1,
\end{array}
$$

and $r=\lambda_{\eta} / \lambda_{\xi}$. In the normal direction, one defines

$$
\left(\bar{\lambda}_{\eta}\right)_{i, j}=\phi_{i, j}\left(\frac{1}{r}\right)\left(\lambda_{\eta}\right)_{i, j}
$$

Alternatives to the switching function presented in (2.9) have been investigated. Caution must be exercised in the selection of a switching variable. If a quantity with the same functional dependence as entropy (i.e., $p / \rho^{\gamma}$ ) is used, sharper shocks can be captured in viscous transonic flows. However, such a choice can result in a loss of accuracy for the surface shear stress, due to the significant variation in the entropy-type variable across the boundary layer. This difficulty can be removed by simply multiplying the scaling factor by a function of the local Mach number of the flow. An acceptable modifying function has proven to be $\left(M_{L} / M_{\infty}\right)^{\beta}$, for some $\beta \geq 1$, where $M_{L}$ is the local Mach number, and $M_{\text {ref }}$ is a reference Mach number (i.e., free-stream value for external flows). It is important to note that the entropy-type function is generally not satisfactory for inviscid flows. In addition, one can consider the sum of two switches, one depending on pressure and the other on temperature so that all thermodynamic changes are taken into account. When introducing the matrix-valued dissipation it will be possible to use separate switches for different characteristic variables.

The treatment of the artificial dissipation must be modified at the boundaries of the physical domain. In the case of the fourth-difference dissipation the standard five point difference stencil must be replaced at the first two interior mesh cells. This means that one-sided or one-sided biased stencils are used at these cells. The dissipative character of the artificial terms is important because it influences both stability and accuracy. For example, if the dissipation is too large at a solid boundary, an artificial boundary layer is created in an inviscid flow, and the effective Reynolds number for a viscous flow is altered. To improve accuracy at the wall boundaries of viscous flows, where gradients are steep 
due to physical boundary layers, the usual fourth-difference stencils are changed in this dissipation model.

Let the total dissipation for a mesh cell, in the direction represented by the index $j$, be denoted by $d_{j}$. For simplicity assume that $\lambda \epsilon^{(4)}=1$. Then,

$$
d_{j}=d f_{j+\frac{1}{2}}-d f_{j-\frac{1}{2}}
$$

where the dissipative flux

$$
d f_{j+\frac{1}{2}}=(\Delta W)_{j+\frac{3}{2}}-2(\Delta W)_{j+\frac{1}{2}}+(\Delta W)_{j-\frac{1}{2}}
$$

and thus

$$
d_{j}=(\Delta W)_{j+\frac{3}{2}}-3(\Delta W)_{j+\frac{1}{2}}+3(\Delta W)_{j-\frac{1}{2}}-(\Delta W)_{j-\frac{3}{2}}
$$

with the index $i$ for $\Delta W$ suppressed for convenience. Consider the first two interior cells adjacent to a solid boundary, as depicted in Figure 1. If

$$
(\Delta W)_{\frac{1}{2}}=(\Delta W)_{\frac{3}{2}}=(\Delta W)_{\frac{3}{2}}
$$

then (2.11) gives

$$
\begin{gathered}
d_{2}=W_{4}-2 W_{3}+W_{2}, \\
d_{3}=W_{5}-4 W_{4}+5 W_{3}-2 W_{2} .
\end{gathered}
$$

These boundary stencils are fairly standard ones, and they result in a nonpositive definite dissipation matrix for the system of difference equations [7]. An alternative form, which has reduced the sensitivity to solid surface normal mesh spacing for turbulent flow calculations without compromising stability or convergence, is given by

$$
(\Delta W)_{\frac{1}{2}}=2(\Delta W)_{\frac{3}{2}}-(\Delta W)_{\frac{5}{2}}
$$

and

$$
\begin{gathered}
d_{2}=W_{4}-3 W_{3}+3 W_{2}-W_{1} \\
d_{3}=W_{5}-4 W_{4}+6 W_{3}-4 W_{2}+W_{1} .
\end{gathered}
$$

This boundary condition is advantageous if the mesh is fine enough to adequately represent the laminar sublayer region of the boundary layer (i.e., at least two mesh points are inside the sublayer). For coarse meshes this treatment can be less accurate than the zeroth-order extrapolation of (2.12). 


\section{The Upwind Connection}

Upwind schemes for solving hyperbolic systems of conservation laws (i.e., Euler equations of gas dynamics) rely upon characteristic theory to determine the direction of propagation of information, and thus, the direction required for one-sided differencing approximations of the spatial derivatives. With such schemes shock waves can be captured without oscillations. Thus, a successful artificial dissipation model for a central-difference scheme should imitate an upwind scheme in the neighborhood of shocks. We now review the connection between these two types of schemes.

Consider the one-dimensional scalar wave equation

$$
u_{t}+a u_{x}=0
$$

with $a$ constant. The first-order upwind scheme can be written as

$$
u_{j}^{n+1}=u_{j}-a \frac{\Delta t}{\Delta x} \begin{cases}u_{j+1}-u_{j}, & a<0 \\ u_{j}-u_{j-1}, & a>0\end{cases}
$$

where all discrete quantities are evaluated at time level $n \Delta t$ unless otherwise denoted. The scheme of (3.1) can be rewritten as

$$
u_{j}^{n+1}=u_{j}-a \frac{\Delta t}{2 \Delta x}\left(u_{j+1}-u_{j-1}\right)+|a| \frac{\Delta t}{2 \Delta x}\left(u_{j+1}-2 u_{j}+u_{j-1}\right),
$$

which now contains a central-difference term and a second-difference dissipation term. Now consider the system

$$
u_{t}+A u_{x}=0
$$

where $u$ is a $\mathrm{N}$-component vector. The system case can be converted to a scalar one by diagonalizing the $N \times N$ matrix $A$ with a similarity transformation

$$
\Lambda=T^{-1} A T
$$

where the columns of $T$ are the right eigenvectors of $A$. After diagonalizing (3.3) and applying the scheme of (3.2), the first-order upwind scheme is given by

$$
u_{j}^{n+1}=u_{j}-A \frac{\Delta t}{2 \Delta x}\left(u_{j+1}-u_{j-1}\right)+|A| \frac{\Delta t}{2 \Delta x}\left(u_{j+1}-2 u_{j}+u_{j-1}\right)
$$

where

$$
|A|=T|\Lambda| T^{-1}, \quad \Lambda=\operatorname{Diag}\left[\left|\lambda_{1}\right| \cdots\left|\lambda_{N}\right|\right]
$$


The generalization to a system of conservation laws is straightforward; namely,

$$
u_{t}+f_{x}=0
$$

with $f$ being a $\mathrm{N}$-component flux vector, and

$$
u_{j}^{n+1}=u_{j}-\frac{\Delta t}{2 \Delta x}\left(f_{j+1}-f_{j-1}\right)+\frac{\Delta t}{2 \Delta x}\left[\left|A_{j+\frac{1}{2}}\right|\left(u_{j+1}-u_{j}\right)-\left|A_{j-\frac{1}{2}}\right|\left(u_{j}-u_{j-1}\right)\right],
$$

where the Jacobian matrix $A=\partial f / \partial u$, and $|A|$ is defined as for (3.4). The matrix $\left|A_{j+\frac{1}{2}}\right|$ can be computed as an arithmetic average or a Roe average. For transonic steady flows the differences are negligible; therefore, we use the simpler arithmetic average. For hypersonic flows Yee [11] found that the Roe average yields better results. For timedependent problems the Roe average also seems to give slightly better results.

\section{Matrix Dissipation Model}

We now extend the scheme given in (3.5) to the two-dimensional equations of fluid dynamics. In particular, consider the transformed Euler equations of (2.2) with the RungeKutta scheme of (2.3). The necessary modification to the contributions for the $\xi$ direction of the artificial dissipation term defined by (2.4) is to substitute $|A|$ for the eigenvalue scaling factor, $\lambda$, in (2.5) and (2.6). For the $\eta$ direction, $\xi$ and $|A|$ are replaced by $\eta$ and $|B|$, respectively. We next define explicitly the form for the matrix $|A|$. Let

$$
\boldsymbol{\Lambda}=\operatorname{Diag}\left[\begin{array}{llll}
\lambda_{1} & \lambda_{2} & \lambda_{3} & \lambda_{3}
\end{array}\right]
$$

with

$$
\begin{gathered}
\lambda_{1}=q+\sqrt{a_{1}^{2}+a_{2}^{2}} c, \quad \lambda_{2}=q-\sqrt{a_{1}^{2}+a_{2}^{2}} c, \quad \lambda_{3}=q, \\
a_{1}=\xi_{x}, \quad a_{2}=\xi_{y}, \quad q=a_{1} u+a_{2} v .
\end{gathered}
$$

Then,

where

$$
\begin{aligned}
|A|= & \left|\lambda_{3}\right| I+\left(\frac{\left|\lambda_{1}\right|+\left|\lambda_{2}\right|}{2}-\left|\lambda_{3}\right|\right)\left[\frac{\gamma-1}{c^{2}} E_{1}+\frac{1}{a_{1}^{2}+a_{2}^{2}} E_{2}\right] \\
& +\frac{\left|\lambda_{1}\right|-\left|\lambda_{2}\right|}{2}\left(\frac{1}{\sqrt{a_{1}^{2}+a_{2}^{2}} c}\right)\left[E_{3}+(\gamma-1) E_{4}\right],
\end{aligned}
$$

$$
E_{1}=\left[\begin{array}{cccc}
\phi & -u & -v & 1 \\
u \phi & -u^{2} & -u v & u \\
v \phi & -u v & -v^{2} & v \\
H \phi & -u H & -v H & H
\end{array}\right]
$$




$$
\begin{aligned}
& E_{2}=\left[\begin{array}{cccc}
0 & 0 & 0 & 0 \\
-a_{1} q & a_{1}^{2} & a_{1} a_{2} & 0 \\
-a_{2} q & a_{2} a_{1} & a_{2}^{2} & 0 \\
-q^{2} & q a_{1} & q a_{2} & 0
\end{array}\right] \\
& E_{3}=\left[\begin{array}{cccc}
-q & a_{1} & a_{2} & 0 \\
-u q & u a_{1} & u a_{2} & 0 \\
-v q & v a_{1} & v a_{2} & 0 \\
-H q & H a_{1} & H a_{2} & 0
\end{array}\right] \\
& E_{4}=\left[\begin{array}{cccc}
0 & 0 & 0 & 0 \\
a_{1} \phi & -a_{1} u & -a_{1} v & a_{1} \\
a_{2} \phi & -a_{2} u & -a_{2} v & a_{2} \\
q \phi & -q u & -q v & q
\end{array}\right]
\end{aligned}
$$

$H$ is the total enthalpy, and $\phi=\left(u^{2}+v^{2}\right) / 2$. Because of the special form of $|A|$ (i.e., each row of $E_{j}$ is a scalar times the first row) for any $\lambda_{1}, \lambda_{2}$, and $\lambda_{3}$, an arbitrary vector $x$ can be multiplied by $|A|$ very quickly. That is, we calculate $\left|A_{j+\frac{1}{2}}\right|\left(u_{j+1}-u_{j}\right)$ directly (see [12]) rather than calculate $\left|A_{j+\frac{1}{2}}\right|$ and multiply a matrix times a vector. The matrix $|B|$ is computed in the same way as $|A|$ by simply replacing $\xi$ with $\eta$.

In practice one cannot choose $\lambda_{1}, \lambda_{2}, \lambda_{3}$ as given above. Near stagnation points $\lambda_{3}$ approaches zero while near sonic lines $\lambda_{1}$ or $\lambda_{2}$ approach zero. A zero artificial viscosity would create numerical difficulties. Hence, we limit these values as

$$
\begin{array}{ll}
\left|\tilde{\lambda}_{1}\right|=\max \left(\left|\lambda_{1}\right|, V_{n} \rho(A)\right), & \rho(A)=|q|+c \sqrt{a_{1}^{2}+a_{2}^{2}}, \\
\left|\tilde{\lambda}_{2}\right|=\max \left(\left|\lambda_{2}\right|, V_{n} \rho(A)\right), & \left|\tilde{\lambda}_{3}\right|=\max \left(\left|\lambda_{3}\right|, V_{\ell} \rho(A)\right),
\end{array}
$$

where the linear eigenvalue $\lambda_{3}$ can be limited differently than the nonlinear eigenvalues. The parameters $V_{n}$ and $V_{\ell}$ have been determined numerically. Various values have been evaluated by comparing their corresponding computed solutions on the basis of the following: 1) Sharpness of shock waves captured (without producing oscillations), 2) Convergence rate of numerical scheme. A good choice for $V_{n}$ and $V_{\ell}$ is between 0.2 and 0.3 .

We have thus far replaced $\lambda_{i+\frac{1}{2}, j}$ in (2.5) and (2.6) by a matrix while leaving the limiters $\epsilon^{(2)}$ and $\epsilon^{(4)}$ as scalars. One can also introduce $\epsilon^{(2)}$ and $\epsilon^{(4)}$ into the diagonal matrix $\Lambda$. This allows different limiters to be chosen for different characteristic variables. For example, the limiter may be based on pressure for the nonlinear waves. However, the pressure is smooth through a contact discontinuity. Hence, a switch based on temperature may be more appropriate for the linear wave. One could also use different mesh scalings, $\phi(r)$, for the linear and nonlinear waves. Also, a smoother cutoff [13] can be introduced. 


\section{The TVD Property}

Consider the one-dimensional scalar conservation law

$$
\frac{\partial}{\partial t}[u(x, t)]+\frac{\partial}{\partial x}[f(u(x, t))]=0
$$

where

$$
-\infty<x<\infty, \quad t \geq 0 .
$$

Let $v(t)=\left\{v_{j}(t)\right\}$ be the approximate solution of (5.1) and consider the semidiscrete equation

$$
\begin{aligned}
\frac{d}{d t} v_{j}(t) & +\frac{1}{2 \Delta x}\left[f_{j+1}-f_{j-1}\right]=\frac{1}{2 \Delta x}\left[Q_{j+\frac{1}{2}} \Delta v_{j+\frac{1}{2}}-Q_{j-\frac{1}{2}} \Delta v_{j-\frac{1}{2}}\right] \\
& -\frac{\kappa^{(4)}}{\Delta x}\left[R_{j+\frac{1}{3}} \Delta^{3} v_{j+\frac{1}{2}}-R_{j-\frac{1}{2}} \Delta^{3} v_{j-\frac{1}{2}}\right]
\end{aligned}
$$

with

$$
\Delta v_{j+\frac{1}{2}}=(\Delta v)_{j+\frac{1}{2}}=v_{j+1}(t)-v_{j}(t)
$$

$\Delta^{3}$ is a third-difference operator defined as

$$
\Delta^{3} v_{j+\frac{1}{2}}=\left(\Delta^{3} v\right)_{j+\frac{1}{2}}=v_{j+2}(t)-3 v_{j+1}(t)+3 v_{j}(t)-v_{j-1}(t) .
$$

The terms on the right hand side of (5.2) represent second- and fourth-difference numerical dissipation terms, with $\kappa^{(4)}$ a constant. Define

$$
s_{j+\frac{1}{2}}=\operatorname{sgn}\left(\Delta v_{j+\frac{1}{2}}\right),
$$

where sgn represents the signum function. Then, a modification of the theorem of Tadmor [14] is given by the following.

Theorem: The semidiscrete scheme of (5.2) is TVD if

(a)

$$
\left[2-s_{j+\frac{1}{2}}\left(s_{j-\frac{1}{2}}+s_{j+\frac{3}{2}}\right)\right] Q_{j+\frac{1}{2}} \geq s_{j+\frac{1}{2}}\left(s_{j+\frac{3}{2}}-s_{j-\frac{1}{2}}\right) \frac{\Delta f_{j+\frac{1}{2}}}{\Delta v_{j+\frac{1}{2}}}
$$

and

(b)

$$
R_{j+\frac{1}{2}}=0 \quad \text { when } \quad s_{j+\frac{3}{3}}-2 s_{j+\frac{1}{2}}+s_{j-\frac{1}{2}} \neq 0 .
$$


Proof: Shifting the indices by one in (5.2) and subtracting (5.2) from the resulting equation, we obtain

$$
\begin{aligned}
\frac{d}{d t} \Delta v_{j+\frac{1}{2}} & +\frac{1}{2 \Delta x}\left[\Delta f_{j+\frac{3}{2}}-\Delta f_{j-\frac{1}{2}}\right] \\
= & \frac{1}{2 \Delta x}\left[Q_{j+\frac{3}{2}} \Delta v_{j+\frac{3}{2}}-2 Q_{j+\frac{1}{2}} \Delta v_{j+\frac{1}{2}}+Q_{j-\frac{1}{2}} \Delta v_{j-\frac{1}{2}}\right] \\
& -\frac{\kappa^{(4)}}{\Delta x}\left[R_{j+\frac{3}{2}} \Delta^{3} v_{j+\frac{3}{2}}-2 R_{j+\frac{1}{2}} \Delta^{3} v_{j+\frac{1}{2}}+R_{j-\frac{1}{2}} \Delta^{3} v_{j-\frac{1}{2}}\right]
\end{aligned}
$$

Multiply (5.3) by $s_{j+\frac{1}{2}}$ and sum over all $j$. Note that $s_{j+\frac{1}{2}}= \pm 1$, so $s_{j+\frac{1}{2}}^{2}=1$, and

$$
s_{j+\frac{1}{2}} \Delta v_{j+\frac{1}{2}}=\left|\Delta v_{j+\frac{1}{2}}\right|
$$

We then obtain

$$
\begin{aligned}
\frac{d}{d t} \sum_{j}\left|\Delta v_{j+\frac{1}{2}}\right|= & -\frac{1}{2 \Delta x} \sum_{j} s_{j+\frac{1}{2}}\left(s_{j-\frac{1}{2}}-s_{j+\frac{3}{2}}\right) \frac{\Delta f_{j+\frac{1}{2}}}{\Delta v_{j+\frac{1}{2}}}\left|\Delta v_{j+\frac{1}{2}}\right| \\
& +\frac{1}{2 \Delta x} \sum_{j} s_{j+\frac{1}{2}}\left(s_{j+\frac{3}{2}}-2 s_{j+\frac{1}{2}}+s_{j-\frac{1}{2}}\right) Q_{j+\frac{1}{2}}\left|\Delta v_{j+\frac{1}{2}}\right| \\
& -\frac{\kappa^{(4)}}{\Delta x} \sum_{j}\left(s_{j+\frac{3}{2}}-2 s_{j+\frac{1}{2}}+s_{j-\frac{1}{2}}\right) R_{j+\frac{1}{2}} \Delta^{3} v_{j+\frac{1}{2}} .
\end{aligned}
$$

We stress that the last term in (5.4) will not help for TVD. Its purpose is to eliminate high frequencies and accelerate convergence to a steady state. Hence, we want this contribution to be zero. This can be accomplished if we demand either

$$
s_{j+\frac{3}{2}}-2 s_{j+\frac{1}{2}}+s_{j-\frac{1}{2}}=0
$$

or

$$
R_{j+\frac{1}{2}}=0
$$

i.e., condition (b). We are left with

$$
\begin{aligned}
\frac{d}{d t}(\mathrm{TV})= & -\frac{1}{2 \Delta x} \sum_{j}\left[-s_{j+\frac{1}{2}}\left(s_{j+\frac{3}{2}}-s_{j-\frac{1}{2}}\right) \frac{\Delta f_{j+\frac{1}{2}}}{\Delta v_{j+\frac{1}{2}}}\right. \\
& \left.-s_{j+\frac{1}{2}}\left(s_{j+\frac{3}{2}}-2 s_{j+\frac{1}{2}}+s_{j-\frac{1}{2}}\right) Q_{j+\frac{1}{2}}\right]\left|\Delta v_{j+\frac{1}{2}}\right|,
\end{aligned}
$$

where TV denotes the total variation as given by

$$
\mathrm{TV}=\sum_{j}\left|\Delta v_{j+\frac{1}{2}}\right|
$$


Thus, a sufficient but not necessary condition that the total variation not increase is that the term of (5.7) in brackets must be positive. This means that

$$
-s_{j+\frac{1}{2}}\left(s_{j+\frac{3}{2}}-2 s_{j+\frac{1}{2}}+s_{j-\frac{1}{2}}\right) Q_{j+\frac{1}{2}} \geq s_{j+\frac{1}{2}}\left(s_{j+\frac{3}{2}}-s_{j-\frac{1}{2}}\right) \frac{\Delta f_{j+\frac{1}{2}}}{\Delta v_{j+\frac{1}{2}}} .
$$

Since $s_{j+\frac{1}{2}}^{2}=1,(5.8)$ is equivalent to condition (a) of the theorem. Defining

$$
\chi_{j}=1-s_{j-\frac{1}{2}} s_{j+\frac{1}{2}}
$$

(5.8) can be written in the form given by Tadmor; namely,

$$
\left(\chi_{j}+\chi_{j+1}\right) Q_{j+\frac{1}{2}} \geq\left(\chi_{j}-\chi_{j+1}\right) \frac{\Delta f_{j+\frac{1}{2}}}{\Delta v_{j+\frac{1}{2}}} .
$$

Several remarks concerning this theorem are in order.

\section{Remarks}

(i) When no extrema are present locally $\left(s_{j-\frac{1}{2}}=s_{j+\frac{1}{2}}=s_{j+\frac{3}{2}}\right)$, both conditions of the theorem are trivially satisfied for all $Q_{j+\frac{1}{2}}$ and $R_{j+\frac{1}{2}}$. For such regions we want $Q_{j+\frac{1}{2}}=O(\Delta x)$ for second-order accuracy and $R_{j+\frac{1}{2}}$ to be chosen so that high frequencies are damped.

(ii) If $s_{j-\frac{1}{2}}=s_{j+\frac{3}{2}}=-s_{j+\frac{1}{2}}$ (a local oscillation at $x_{j+\frac{1}{2}}$ ), we require

$$
Q_{j+\frac{1}{2}} \geq 0, \quad R_{j+\frac{1}{2}}=0 .
$$

(iii) If $s_{j-\frac{1}{2}}=s_{j+\frac{1}{2}}=-s_{j+\frac{3}{2}}$ (a local extremum at $x_{j+1}$ ),

(iv) If $s_{j-\frac{1}{2}}=-s_{j+\frac{1}{2}}=-s_{j+\frac{3}{2}}$ (a local extremum at $x_{j}$ ),

$$
Q_{j+\frac{1}{2}} \geq \frac{-\Delta f_{j+\frac{1}{2}}}{\Delta v_{j+\frac{1}{2}}}, \quad R_{j+\frac{1}{2}}=0 .
$$

$$
Q_{j+\frac{1}{2}} \geq \frac{\Delta f_{j+\frac{1}{2}}}{\Delta v_{j+\frac{1}{2}}}, \quad R_{j+\frac{1}{2}}=0 .
$$

It follows from these inequalities that $Q_{j+\frac{1}{2}}$ can be negative. As far as total variation is concerned, central differences are not nondissipative. That is, they can either increase or 
decrease the total variation. In cases where central differences decrease the total variation, $Q_{j+\frac{1}{2}}$ can be negative. For systems of equations, especially in multidimensions, this behavior can sometimes lead to difficulties. Hence, we demand the stronger condition that

$$
Q_{j+\frac{1}{2}} \geq\left|\frac{\Delta f_{j+\frac{1}{2}}}{\Delta v_{j+\frac{1}{2}}}\right|
$$

For systems this condition is replaced by

$$
Q_{j+\frac{1}{2}} \geq\left|A_{j+\frac{1}{2}}\right|
$$

where $A=\partial f / \partial u$, and the average at $j+\frac{1}{2}$ is constructed by the technique of Roe [2].

\section{Flux Limiters}

In this section switching functions are introduced that force the scheme to automatically satisfy the inequalities presented in Section $V$. These switches are required to be smooth so that limit cycles are not experienced when marching in time to obtain a steadystate solution. The discrete switching functions are defined as

$$
\begin{aligned}
& Q_{j+\frac{1}{2}}=\psi \cdot\left|a_{j+\frac{1}{2}}\right| \text { with } 0 \leq \psi \leq 1 \\
& R_{j+\frac{1}{2}}=\Gamma \cdot\left|a_{j+\frac{1}{2}}\right|
\end{aligned}
$$

where $\psi=1$ near extrema, so that the inequalities are satisfied, and $\psi=O(\Delta x)$ in smooth regions of the flow field. Conversely, $\Gamma=0$ at extrema, and $\Gamma=1$ in the smooth regions.

The functions $\psi$ and $\Gamma$ of (6.1) can be defined in terms of a limiter function. Let

$$
r=\frac{v_{j}-v_{j-1}}{v_{j+1}-v_{j}}=\frac{\Delta v_{j-\frac{1}{2}}}{\Delta v_{j+\frac{1}{2}}}
$$

The van Leer flux limiter is given by

$$
\varphi_{j}(r)=\frac{r+|r|}{1+|r|}= \begin{cases}\frac{2 r}{1+r}, & r>0 \\ 0, & r \leq 0\end{cases}
$$

From Sweby [14] it is straightforward to see that for any flux limiter

$$
\psi_{j}(r)=1-\varphi_{j}(r)
$$


Since we want $\psi_{j}(r)$ to be positive, this relation is redefined as

$$
\psi_{j}(r)=\left|1-\varphi_{j}(r)\right|
$$

Then, for the van Leer limiter,

$$
\psi_{j}(r)=\left|\frac{1-r}{1+|r|}\right|= \begin{cases}\frac{|1-r|}{1+r}, & r>0 \\ 1, & r \leq 0\end{cases}
$$

so $0 \leq \psi_{j} \leq 1$ and define

$$
\psi_{j+\frac{1}{2}}=\max \left(\psi_{j}, \psi_{j+1}\right)
$$

We now show that the inequality (5.8) is satisfied with this $\psi$. By the first remark of Section $V$, the inequality is satisfied when $s_{j-\frac{1}{2}}=s_{j+\frac{1}{2}}=s_{j+\frac{3}{2}}$, and so in this case we only need $Q=O(\Delta x)$. Since $r>0$ in this case,

$$
\psi_{j}(r)=\frac{|1-r|}{1+r}
$$

In addition, for smooth regions of the flow field,

$$
r=1+O(\Delta x)
$$

and thus

$$
\psi_{j+\frac{1}{2}}=O(\Delta x), \quad Q_{j+\frac{1}{2}}=O(\Delta x) .
$$

Next, consider the case $s_{j-\frac{1}{2}}=-s_{j+\frac{1}{2}}$. This implies $r_{j}<0$, and so, $\psi_{j}=1$. Moreover,

$$
s_{j-\frac{1}{2}}=-s_{j+\frac{1}{2}} \Rightarrow \psi_{j}=1 \Rightarrow \psi_{j+\frac{1}{2}}=1 \text {. }
$$

Similarly, if $s_{j+\frac{3}{2}}=-s_{j+\frac{1}{2}}$,

$$
s_{j+\frac{1}{2}}=-s_{j+\frac{3}{2}} \Rightarrow \psi_{j+1}=1 \Rightarrow \psi_{j+\frac{1}{2}}=1 .
$$

It also follows, using (6.1), that the inequalities in (ii) and (iii) of the remarks of Section $\mathrm{V}$ are satisfied. Also, for these two cases, setting $\Gamma_{j+\frac{1}{2}}=1-\psi_{j+\frac{1}{2}}$ guarantees that $\Gamma_{j+\frac{1}{2}}=0$.

$$
\Gamma_{j+\frac{1}{2}}=1-\max \left(\psi_{j}, \psi_{j+1}\right)
$$


The flux limiters $\psi$ and $\Gamma$ can be connected to those used in upwind schemes. Sweby [15] considers an upwind Lax-Wendroff scheme. In particular, for the one-dimensional wave equation

$$
u_{t}+a u_{x}=0
$$

the numerical solution is obtained with

$$
u_{j}^{n+1}=u_{j}-\nu\left(u_{j}-u_{j-1}\right)-\Delta_{-}\left[\frac{\nu(1-\nu)}{2} \varphi_{j}\left(u_{j+1}-u_{j}\right)\right]
$$

where

$$
\nu=a \frac{\Delta t}{\Delta x}, \quad 0 \leq \varphi(r) \leq 2, \quad \frac{\varphi(r)}{r}=\varphi\left(\frac{1}{r}\right)
$$

and the backward difference operator $\Delta_{-}$is defined by

$$
\Delta_{-} u_{j}=u_{j}-u_{j-1}
$$

If (6.2) is rewritten as a central-difference scheme, then

$$
\begin{aligned}
u_{j}^{n+1}= & u_{j}-\frac{\nu}{2}\left(u_{j+1}-u_{j-1}\right)+\frac{\nu}{2}\left[\left(1-\varphi_{j}\right)\left(u_{j+1}-u_{j}\right)-\left(1-\varphi_{j-1}\right)\left(u_{j}-u_{j-1}\right)\right] \\
& +\frac{\nu^{2}}{2}\left[\varphi_{j}\left(u_{j+1}-u_{j}\right)-\varphi_{j-1}\left(u_{j}-u_{j-1}\right)\right]
\end{aligned}
$$

By dropping the $\nu^{2}$ term in (6.3) and changing to a semidiscrete formulation, we get

$$
\begin{aligned}
\frac{d}{d t} u_{j}= & -\frac{\nu}{2 \Delta t}\left(u_{j+1}-u_{j-1}\right) \\
& +\frac{\nu}{2 \Delta t}\left[\left(1-\varphi_{j}\right)\left(u_{j+1}-u_{j}\right)-\left(1-\varphi_{j-1}\right)\left(u_{j}-u_{j-1}\right]\right.
\end{aligned}
$$

If

$$
\varphi_{j}=1-\psi_{j+\frac{1}{2}}
$$

then the second-difference dissipation term has the same form as the one presented in Section V.

To complete the connection between the limiters for the central-difference and upwind schemes, we compare their behavior. For the central-difference case,

$$
0 \leq \psi \leq 1, \quad 0 \leq \varphi \leq 1
$$

whereas for the upwind case, $0 \leq \varphi \leq 2$. Furthermore, for the central-difference limiter,

$$
\psi\left(\frac{1}{r}\right)=\psi(r), \quad \varphi\left(\frac{1}{r}\right)=\varphi(r)
$$


means that it does not matter if $\Delta u_{j+\frac{1}{2}}>\Delta u_{j-\frac{1}{2}}$ or $\Delta u_{j+\frac{1}{2}}<\Delta u_{j-\frac{1}{2}}$ (i.e., the sign of $a$ does not affect the scheme), as opposed to the upwind limiter where

$$
\frac{\varphi(r)}{r}=\varphi\left(\frac{1}{r}\right)
$$

In Figure 2 the Sweby diagram for the upwind van Leer flux limiter and the centraldifference van Leer flux limiter is shown. For $r<1$, the limiters are the same. For $r>1$, the upwind version continues until $\varphi=2$, while the central-difference version returns to zero. In Sweby's paper the flux limiters are not allowed to decrease when $r>1$. However, there is no difficulty from such behavior. In both cases $\varphi(r)=0$ when $r \leq 0$ so that the limiter is turned off for extrema. We note that other limiters besides that of van Leer can be used to get switches for central-difference schemes.

For systems of equations we use a scalar limiter. Using the matrix form of the dissipation it is easy to implement different limiters for different characteristic variables.

In terms of the pressure the switch becomes

$$
\psi_{j}=\frac{1-r}{1+|r|}, \quad r=\frac{\Delta p_{j-\frac{1}{2}}}{\Delta p_{j+\frac{1}{2}}}, \quad a>0
$$

or

with

$$
\psi_{j}=\frac{\left|p_{j+1}-p_{j}\right|-\left(p_{j}-p_{j-1}\right) \operatorname{sgn}\left(p_{j+1}-p_{j}\right)}{\left|p_{j+1}-p_{j}\right|+\left|p_{j}-p_{j-1}\right|+\epsilon}, \quad a>0
$$

$$
\psi_{j+\frac{1}{2}}=\max \left(\psi_{j}, \psi_{j+1}\right)
$$

and $\epsilon=O\left(\Delta x^{2}\right)$ to prevent a zero denominator for constant pressure regions. Consider also the wave speed $a<0$. Then,

$$
\psi_{j}=\frac{p_{j+1}-2 p_{j}+p_{j-1}}{\left|p_{j+1}-p_{j}\right|+\left|p_{j}-p_{j-1}\right|+\epsilon} \begin{cases}\operatorname{sgn}\left(p_{j+1}-p_{j}\right), & a>0 \\ \operatorname{sgn}\left(p_{j-1}-p_{j}\right), & a<0 .\end{cases}
$$

We use a conservative approach and take

$$
\psi_{j}=\frac{\left|p_{j+1}-2 p_{j}+p_{j-1}\right|}{\left|p_{j+1}-p_{j}\right|+\left|p_{j}-p_{j-1}\right|+\epsilon}
$$

Notice that this switch is very similar to (2.9) for the original dissipation model of the Runge-Kutta scheme. There is only a minor change in the denominator. However, with this change and the factor $1 / 2$ in front of the second-difference dissipation term, the scalar 
equation becomes first-order upwind near shocks. In the case of the original $\psi_{j}$ we find that $\psi_{j} \simeq .05$ near shock waves in transonic flows. One may require different parameters for the Runge-Kutta scheme to ensure stability.

We now no longer have a free parameter for the second-difference dissipation. We also usually use $\Gamma=1-2 \psi$ so that the fourth-difference dissipation is cut off for $\psi \geq 1 / 2$. The only free parameter is the coefficient $\kappa^{(4)}$ of the fourth-difference term.

\section{Numerical Algorithm}

The majority of the numerical results presented in this paper were obtained with a Navier-Stokes code developed by the authors, which is based on the explicit multistage time-stepping schemes of [3] and [16]. This class of schemes is currently in widespread use for solving the Euler equations. In references [17-20], these schemes were extended to allow solution of the compressible Navier-Stokes equations. Significant improvements in numerical efficiency were introduced in [9], [10], and [21]. In the code of Swanson and Turkel a cell-centered, finite-volume method is employed to obtain centered type difference approximations for the flow equations. Such a method provides flexibility in treating arbitrary geometries and different grid topologies, since no special treatment is required in the vicinity of singular points or lines. The scheme is second-order accurate in space for sufficiently smooth meshes (see [21-22] for definition of sufficiently smooth). A modified five stage Runge-Kutta scheme is used for the time integration to obtain a steady-state solution. There is a weighted evaluation of the artificial dissipation terms on the first, third, and fifth stages, which provides a large parabolic stability limit. The physical viscous terms are computed on the first stage only and frozen for the remaining ones; this does not compromise the stability characteristics of the scheme. The spatial and temporal differencing are decoupled. Thus, the numerical algorithm is independent of time step and amenable to steady-state convergence acceleration techniques. These methods include local time stepping (a preconditioning for the system of difference equations), variable coefficient implicit residual smoothing, and multigrid. Implicit residual smoothing is just a mathematical step, applied at each stage of the explicit scheme, to extend the local stability range. The multigrid method involves cycling through a sequence of successively coarser grids and relying upon effective high frequency damping for rapid removal of the errors in the fine grid solution. 


\section{Applications}

Two airfoil flow problems are considered here to demonstrate the benefits of using a central-difference scheme with a matrix numerical dissipation model. The first problem concerns inviscid flow over an NACA 0012 airfoil. At a free-stream Mach number $\left(\mathrm{M}_{\infty}\right)$ of 0.8 and angle of attack $(\alpha)$ of 1.25 degrees, a fairly strong transonic shock wave occurs on the upper surface, while a much weaker one appears on the lower surface. On representative inviscid meshes, schemes based on central differencing capture the upper surface shock reasonably well, but they smear significantly the lower surface shock. The second problem involves transonic turbulent flow over a RAE 2822 airfoil. In this case the free-stream Mach number is 0.73 , the angle of attack is 2.79 degrees, and the Reynolds number based on chord $\left(\mathrm{Re}_{\infty}\right)$ is $6.5 \times 10^{6}$. For such transonic viscous flows small differences in the shock strength can result in noticeable changes in the lift and drag of the airfoil. Thus, both of these problems can provide a reasonable measure of the performance of the artificial dissipation model, especially near shock waves.

A C-type mesh consisting of 224 cells around the airfoil (160 cells on the airfoil) and 32 cells normal to the airfoil was used for the first problem. The outer boundary of the finite domain was placed 20 chords away from the airfoil, so as to not produce significant effects on the solutions (see [23]). The normal mesh spacing at the surface was approximately 0.01 chords. The mesh was clustered at the leading and trailing edges of the airfoil in order to improve resolution of the steep gradients occurring in these regions. In Figure 3 the surface pressure distributions computed with the scalar and matrix dissipation models are shown. There is a discernible improvement in the sharpness of the shock waves using the matrix model, especially for the one on the lower airfoil surface. The lift and drag coefficients obtained using the two models, along with the values from a high density mesh calculation, are compared in Table I. The values associated with the matrix model nearly match those corresponding to the fine mesh solution. The convergence histories in terms of number of multigrid cycles are also displayed in Figure 3. Convergence is measured by the logarithm of the root mean square of the residual of the continuity equation. The convergence rates obtained with the scalar and matrix models are 0.819 and 0.888 , respectively. The matrix model result required about $55 \mathrm{cpu} \mathrm{sec}$ on a Cray II computer for 125 multigrid cycles with the solution grid. This processing time represents a 15 percent increase in the time needed compared with the scalar dissipation model. It may be possible to improve the convergence rate with the matrix model by altering the 
coefficients of the time-stepping scheme and/or the type of implicit residual smoothing operator employed.

The following set of C-type meshes were used in solving the second problem with the two dissipation models: 1) $160 \times 32$ with 128 cells on the airfoil, 2) $320 \times 64$ with 256 cells on the airfoil, 3) $640 \times 128$ with 512 cells on the airfoil. Each successively coarser mesh was generated by eliminating every other mesh line in both coordinate directions of the finer mesh. Again the outer boundary of the domain was located 20 chords from the airfoil. The normal spacing at the surface for the finest mesh was $7.5 \times 10^{-6}$ chords. In Figure 4 a comparison is made between the experimental data of [24] and the predicted distributions of pressure, upper surface skin friction, and upper surface boundary-layer displacement thickness obtained on the $160 \times 32$ mesh. The skin-friction coefficient is the wall shear stress nondimensionalized by the dynamic pressure at the edge of the viscous layer. Velocity profiles (scaled by the boundary-layer edge velocity $u_{e}$ ) at two stations on the upper airfoil surface are also presented in Figure 4. There is marked improvement in the results obtained with the matrix model, as verified by their reasonable agreement with the data. The convergence histories given in Figure 4 indicate that the final residual level is somewhat higher with the matrix model. However, the terminal rate of convergence is nearly the same with both dissipation models. Finally, Figures 5 and 6 show the pressure and skin-friction distributions computed on the coarse mesh with second-order upwind and "third-order" upwind biased forms of Roe's scheme [2], which has been shown to have low levels of dissipation. The pressures obtained with the "third-order" form are very close to those calculated with the matrix model. The upwind skin-friction solutions exhibit slightly better agreement with the experimental data upstream of the shock.

Numerical results for the two finer meshes are presented in Figures 7 and 8 . For the $320 \times 64$ mesh, a slightly stronger shock is predicted using the matrix dissipation model. Otherwise, the solutions determined with the different dissipation models are nearly the same. The character of the convergence behavior with the two models is about the same as for the coarse mesh. On the finest mesh, the application of the models gives essentially the same results. In Figure 9 the variation of the computed lift and total drag coefficients with the reciprocal of the number of mesh cells is plotted for each dissipation model. The curves corresponding to the matrix model are nearly linear. A linear curve indicates second-order accuracy for the full range of meshes being considered. The numerical values for the components of the drag coefficient (form and friction contributions) along with the 
lift coefficient are given for all the viscous computations in Table II. Notice that the values obtained with the matrix model on the $320 \times 64$ grid are very close to those calculated with the scalar model on the $640 \times 128$ grid. Such a reduction in the mesh required for acceptable accuracy is highly desirable, especially for three-dimensional flow problems. Some applications to three-dimensional viscous flows are presented in Turkel and Vatsa [25]. It should be emphasized that accurate prediction of lift and drag is very important in the design of aircraft, and thus, a good estimate of these quantities for an infinitely fine mesh is needed.

In [26] it is shown that the TVD switch (6.4) allows converged solutions for hypersonic flow where the standard switch (2.9) does not.

\section{Concluding Remarks}

We have thus shown that the second-difference artificial dissipation is equivalent to using a flux limiter, and hence, central-difference schemes are not any more "artificial" than upwind schemes. The central-difference scheme is slightly more dissipative for two reasons. First, $\psi_{j+\frac{1}{2}}=\max \left(\psi_{j}, \psi_{j+1}\right)$ while for an upwind scheme $\psi_{j+1}$ is equal to either $\psi_{j}$ or $\psi_{j+1}$, depending on the direction of the wind. Second, we insist that $\psi_{j}$ be positive (i.e., $\varphi_{j} \leq 1$ ) while upwind limiters allow $\varphi_{j}>1$ (i.e., in some cases we can have negative viscosity but still be TVD). However, to compensate for this slight increase in dissipation central-difference schemes are simpler to program and require less computer time per time step, and work well with multigrid acceleration techniques.

In addition the central-difference schemes have a free parameter in conjunction with the fourth-difference dissipation. This dissipation is needed to approach a steady state and has nothing to do with TVD properties. In fact the fourth-difference contribution is set equal to zero near local extrema. For time-dependent flows one can set this dissipation identically to zero. On the other hand TVD properties do not necessarily imply a rapid convergence to the steady state.

In summary, a formulation for a numerical dissipation model that makes a centraldifference scheme closely resemble an upwind scheme near flow discontinuities has been described. A theorem has been proven that gives sufficient requirements for this type of dissipation model to satisfy the TVD property for a scalar equation. Flux limiter functions have been presented for this form of dissipation model. For a system of equations a matrixvalued dissipation is introduced. Solutions of the Euler and Navier-Stokes equations for 
airfoil flows have been obtained using the matrix dissipation model. These results have demonstrated noticeable improvements in accuracy in smooth regions of the flow field as well as near shock waves. There is a 15 percent increase in computational time for explicit multistage schemes when this model is used. However, the improved accuracy has permitted a significant reduction in the number of mesh points required. Such behavior of a scheme can have a dramatic effect on the necessary mesh size for three-dimensional flow calculations.

Finally, it is important to emphasize the different principal objectives associated with matrix-valued dissipation and the TVD switch. The purpose of the matrix form of the numerical dissipation model is to apply the appropriate scaling of the dissipation in each flow equation, yielding a reduction in the amount of dissipation being introduced and improved accuracy. The TVD switch plays a somewhat opposite role in that it causes more dissipation to be added in order to prevent overshoots, and thus, allows convergence and provides robustness in solving high speed flow problems. The combination of the two should give more dissipation near shocks and less dissipation in smooth regions, hence giving better accuracy in all regions.

\section{Acknowledgement}

The authors would like to express their appreciation to Dr. Venkatakrishnan for providing numerical solutions obtained with the upwind scheme of Roe. 


\section{References}

1. van Leer, B., "Flux Vector Splitting for the Euler Equations," Eighth International Conference on Numerical Methods in Fluid Dynamics, Springer Verlag Lecture Notes in Physics, No. 170, edited by E. Krause, 1982, pp. 507-512.

2. Roe, P. L., "Approximate Riemann Solvers, Parameter Vectors, and Difference Schemes," Journal of Computational Physics, Vol. 43, 1981, pp. 357-372.

3. Jameson, A., Schmidt, W., and Turkel, E., "Numerical Solutions of the Euler Equations by Finite Volume Methods Using Runge-Kutta Time-Stepping Schemes," AIAA Paper 81-1259, June 1981.

4. Jameson, A. and Baker, T. J., "Multigrid Solution of the Euler Equations for Aircraft Configurations," AIAA Paper 84-0093, January 1984.

5. Deese, J. E. and Agarwal, R. K., "Calculations of Axisymmetric Inlet Flowfield Using the Euler Equations," AIAA Paper 83-1853, July 1983.

6. Subramanian, S. V. and Bozzola, R., "Application of Runge-Kutta Time Marching Scheme for the Computation of Transonic Flows in Turbomachines," AIAA Paper 851332, July 1985.

7. Pulliam, T. H., "Artificial Dissipation for the Euler Equations," AIAA Journal, Vol. 24, pp. 1931-1940, 1986.

8. Jameson, A. and Yoon, S., "Lower-Upper Implicit Schemes with Multiple Grids for the Euler Equations," AIAA Journal, Vol. 25, July 1987, pp. 929-935.

9. Swanson, R. C. and Turkel, E., "Artificial Dissipation and Central Difference Schemes for the Euler and Navier-Stokes Equations," AIAA Paper 87-1107, AIAA 8th Computational Fluid Dynamics Conference, Honolulu, Hawaii, June 9-11, 1987.

10. Martinelli, L. and Jameson, A., "Validation of a Multigrid Method for the Reynolds Averaged Equations," AIAA Paper 88-0414, January 1988.

11. Yee, H. C., "Upwind and Symmetric Shock-Capturing Schemes, NASA TM 89464, 1987.

12. Turkel, E., "Improving the Accuracy of Central Difference Schemes," Springer-Verlag Lecture Notes in Physics, 11th International Conference on Numerical Methods in Fluid Dynamics, Vol. 323, 1988, pp. 586-591 (see also NASA CR 181712).

13. Harten, A., "High Resolution Schemes for Hyperbolic Conservation Laws," Journal of Computational Physics, Vol. 49, 1983, pp. 357-393. 
14. Tadmor, E., "Convenient Total Variation Diminishing Conditions for Nonlinear Difference Schemes," SIAM Journal of Numerical Analysis, Vol. 25, No. 5, October 1988, pp. 1002-1014.

15. Sweby, P. K., "High Resolution Schemes Using Flux Limiters for Hyperbolic Conservation Laws," SIAM Journal of Numerical Analysis, Vol. 21, No. 5, October 1984, pp. 995-1011.

16. Jameson, A., “Multigrid Algorithms for Compressible Flow Calculations," MAE Report 1743, Princeton University, Text of lecture given at 2nd European Conference on Multigrid Methods, Cologne, October 1985.

17. Swanson, R. C. and Turkel, E., "A Multistage Time-stepping Scheme for the NavierStokes Equations," AIAA Paper 85-0035, January 1985.

18. Agarwal, R. K. and Deese, J. E., "Computation of Transonic Viscous Airfoil, Inlet, and Wing Flowfields," AIAA Paper 84-1551, June 1984.

19. Martinelli, L., Jameson, A., and Grasso, F., "A Multigrid Method for the NavierStokes Equations," AIAA Paper 86-0208, January 1986.

20. Vatsa, V. N., "Accurate Solutions for Transonic Viscous Flow Over Finite Wings," Journal of Aircraft, Vol. 24, No. 6, June 1987, pp. 377-385.

21. Radespiel, R. and Swanson, R. C., "An Investigation of Cell Centered and Cell Vertex Multigrid Schemes for the Navier-Stokes Equations," AIAA Paper 89-0548, January 1989.

22. Turkel, E., "Accuracy of Schemes Using Nonuniform Meshes for Compressible Fluid Flows," Applied Numerical Mathematics, Vol. 2, 1986, pp. 529-550.

23. Thomas, J. L. and Salas, M. D., "Far-Field Boundary Conditions for Transonic Lifting Solutions to the Euler Equations," AIAA Paper 85-0020, January 1985.

24. Cook, P. H., McDonald, M. A., and Firmin, M. C. P., "AEROFOIL RAE 2822 Pressure Distributions, and Boundary Layer and Wake Measurements," AGARD Advisory Report No. 138, May 1979.

25. Turkel, E. and Vatsa, V. N., "Effect of Artificial Viscosity on Three-Dimensional Flow Solutions," AIAA Paper 90-1445, June 1990.

26. Decker, N. and Turkel, E., "Multigrid Methods for Compressible Fluid Dynamics," Proceedings of Third International Conference on Hyperbolic Problems, Uppsala, Sweden, June 1990. 


\section{Table I}

Lift and drag coefficients for NACA 0012 airfoil,

$$
\mathrm{M}_{\infty}=0.8, \alpha=1.25^{\circ}
$$

\begin{tabular}{ccc}
\hline \hline Case & $\mathrm{c}_{\mathrm{l}}$ & $\mathrm{c}_{\mathrm{d}}$ \\
\hline $\begin{array}{c}\text { Scalar dissipation model } \\
(224 \times 32 \text { mesh })\end{array}$ & 0.3628 & 0.0231 \\
$\begin{array}{c}\text { Matrix dissipation model } \\
(224 \times 32 \text { mesh })\end{array}$ & 0.3591 & 0.0227 \\
$\begin{array}{c}\text { Scalar dissipation model } \\
(640 \times 64 \text { mesh })\end{array}$ & 0.3577 & 0.0228 \\
\hline
\end{tabular}

$c_{1}$ - lift coefficient

$c_{d}-$ drag coefficient 


\section{Table II}

Lift and drag coefficients for RAE 2822 airfoil,

$$
\mathrm{M}_{\infty}=0.73, \alpha=2.79^{\circ}, \operatorname{Re}_{\infty}=6.5 \times 10^{6}
$$

\begin{tabular}{|c|c|c|c|c|}
\hline \multicolumn{5}{|c|}{ Scalar dissipation model } \\
\hline Mesh & $c_{1}$ & $c_{d_{p}}$ & $c_{d_{f}}$ & $c_{d_{\text {tot }}}$ \\
\hline $160 \times 32$ & 0.8081 & 0.0128 & 0.0045 & 0.0173 \\
\hline $320 \times 64$ & 0.8395 & 0.0120 & 0.0054 & 0.0174 \\
\hline $640 \times 128$ & 0.8544 & 0.0123 & 0.0055 & 0.0178 \\
\hline \multicolumn{5}{|c|}{ Matrix dissipation model } \\
\hline $160 \times 32$ & 0.8296 & 0.0124 & 0.0050 & 0.0174 \\
\hline $320 \times 64$ & 0.8514 & 0.0123 & 0.0055 & 0.0178 \\
\hline $640 \times 128$ & 0.8588 & 0.0124 & 0.0055 & 0.0179 \\
\hline \multicolumn{5}{|c|}{ Second-order upwind } \\
\hline $160 \times 32$ & 0.8176 & 0.0119 & 0.0054 & 0.0173 \\
\hline \multicolumn{5}{|c|}{ "Third-order" upwind biased } \\
\hline $160 \times 32$ & 0.8220 & 0.0124 & 0.0054 & 0.0178 \\
\hline
\end{tabular}

$c_{1}$ - lift coefficient

$c_{d_{p}}$ - pressure drag coefficient

$c_{d_{f}}$ - friction drag coefficient

$c_{d_{\text {tot }}}$ - total drag coefficient 


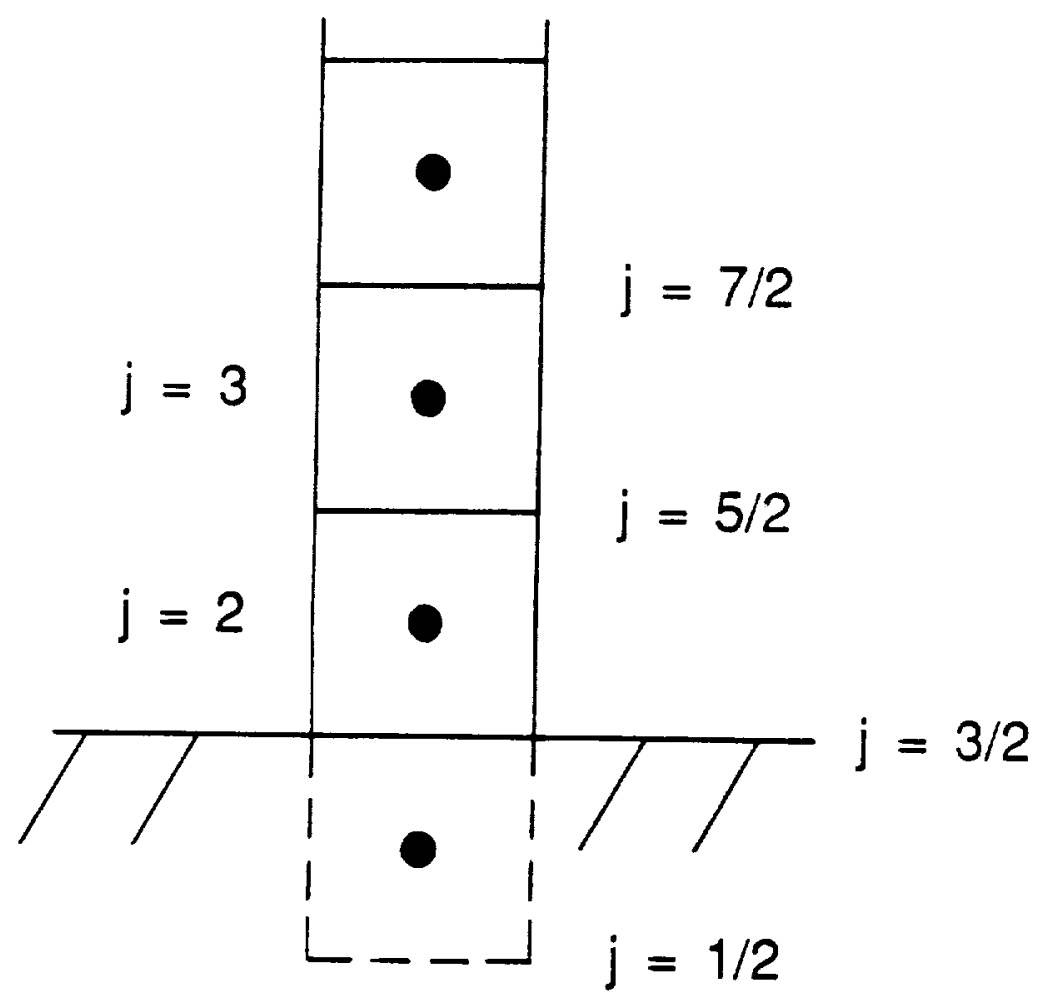

Figure 1 Boundary point dissipation 


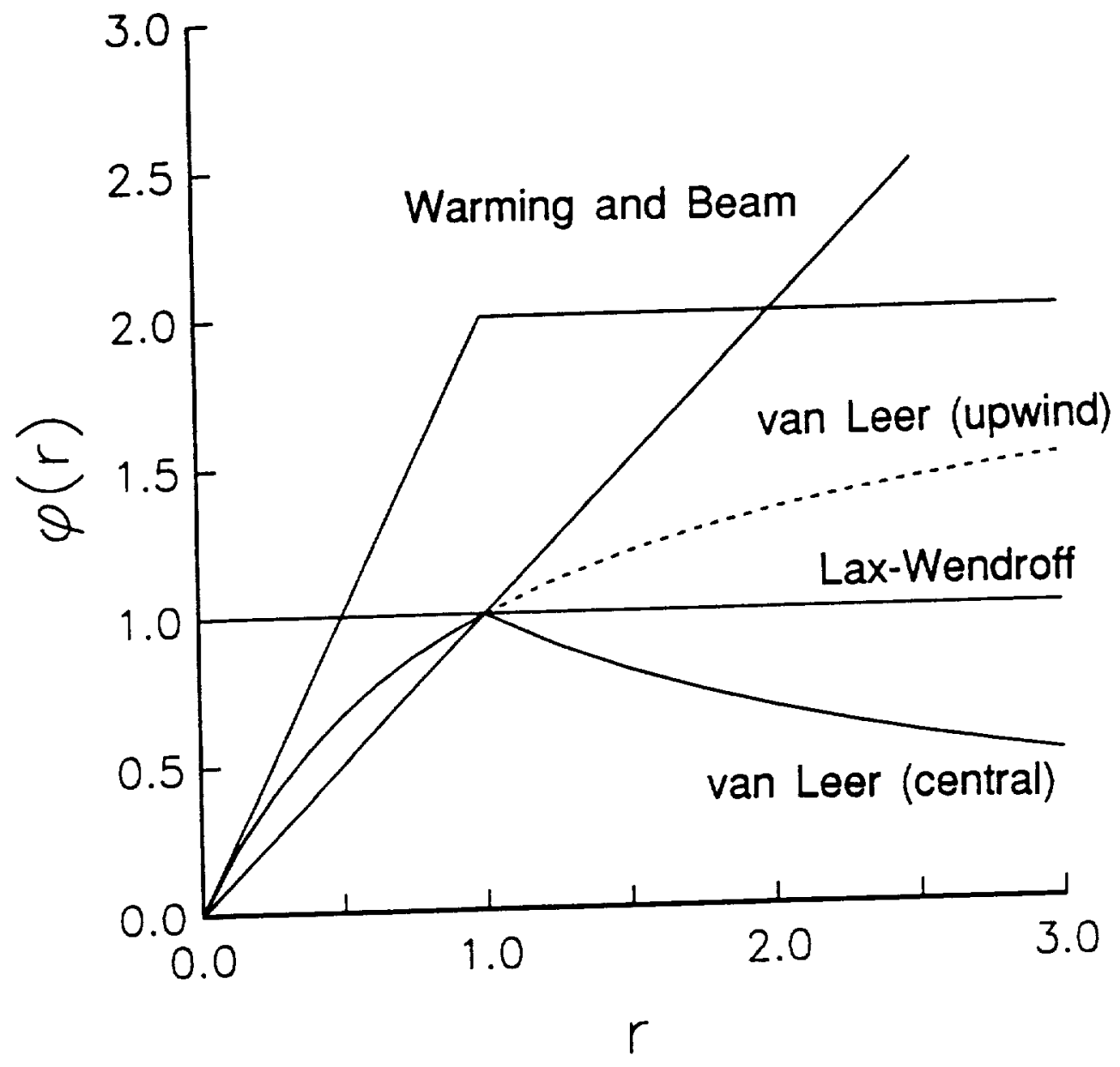

Figure 2 Sweby diagram 


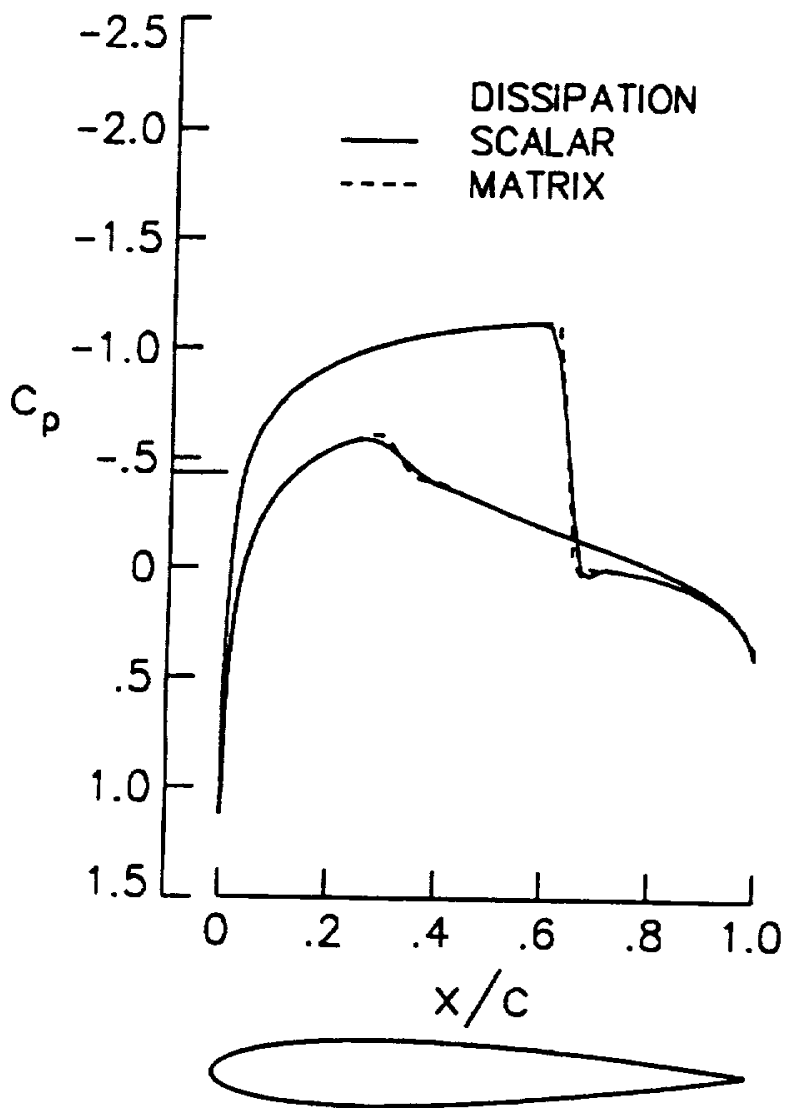

(a)

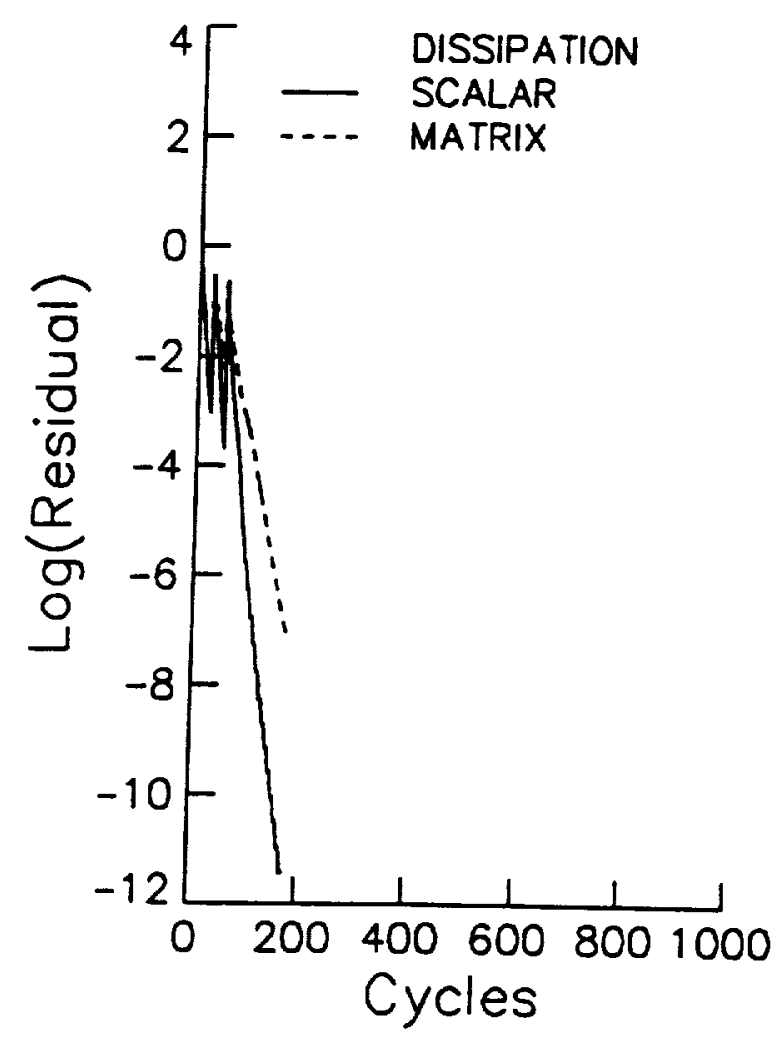

(b) Figure 3 Central-difference scheme results for inviscid flow over NACA 0012 airfoil
$\left(M_{\infty}=0.80, \alpha=1.25\right.$ degrees):

(a) Surface pressure coefficient, (b) Convergence history 


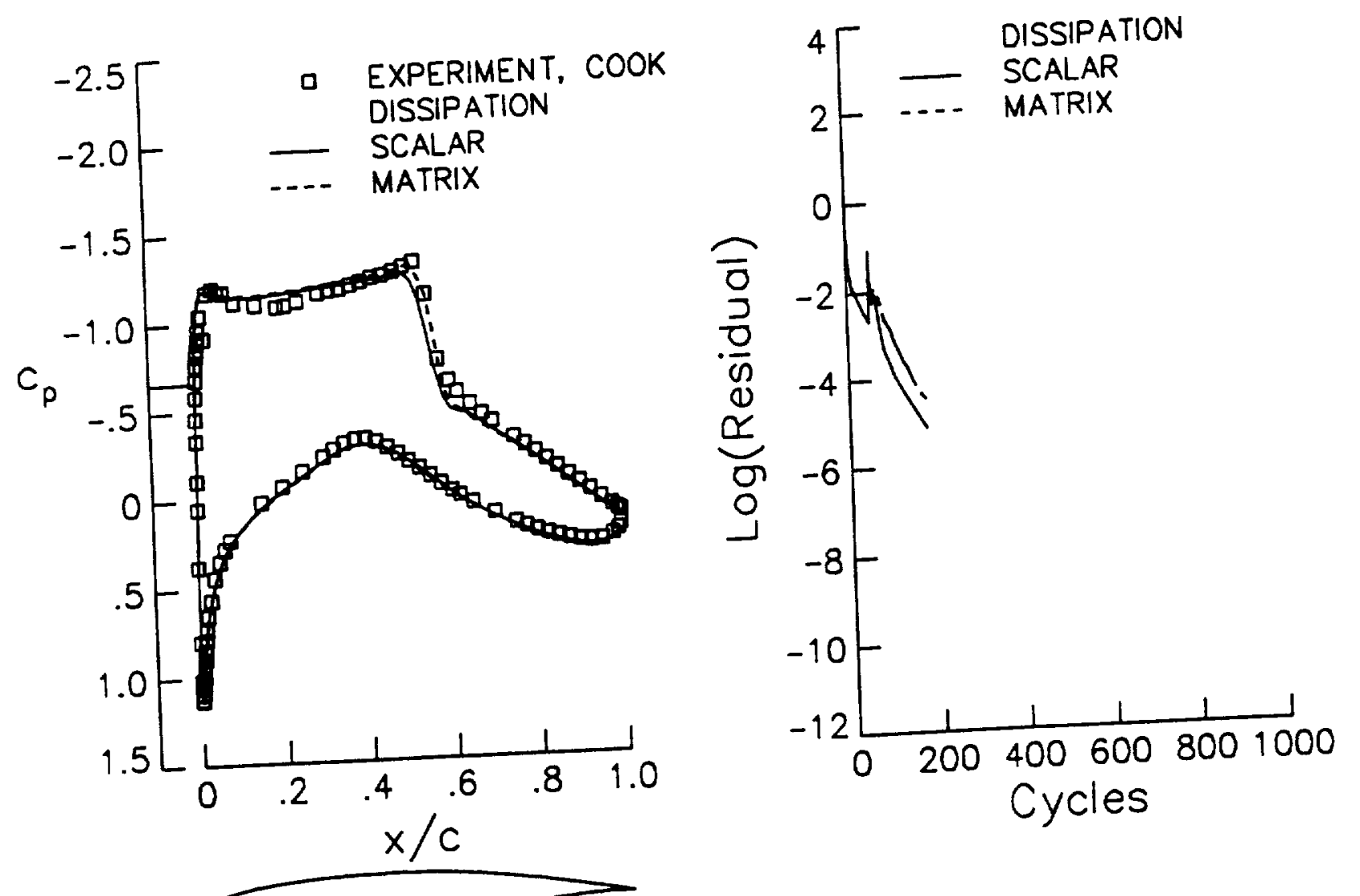

(a)

(b)

Figure 4 Central-difference scheme results for turbulent flow over RAE 2822 airfoil $\left(160 \times 32\right.$ mesh, $M_{\infty}=0.73, \alpha=2.79$ degrees, $\left.\operatorname{Re}_{\infty}=6.5 \times 10^{6}\right)$ :

(a) Surface pressure coefficient, (b) Convergence history 

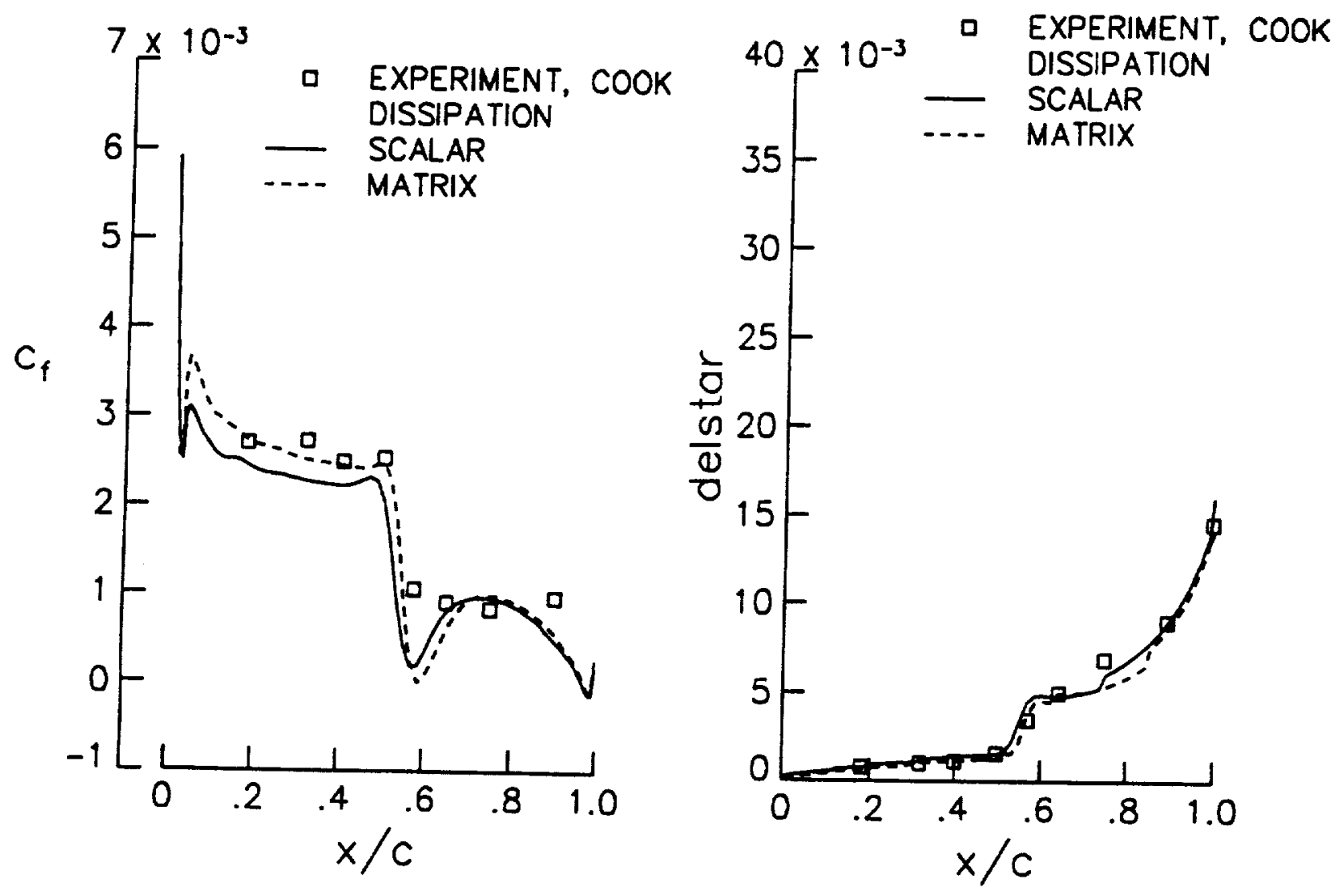

(c)

(d) Figure $4 c-4 d$ Upper surface skin-friction coefficient $\left(c_{f}\right)$ and boundary-layer displacement
thickness $(160 \times 32$ mesh) 

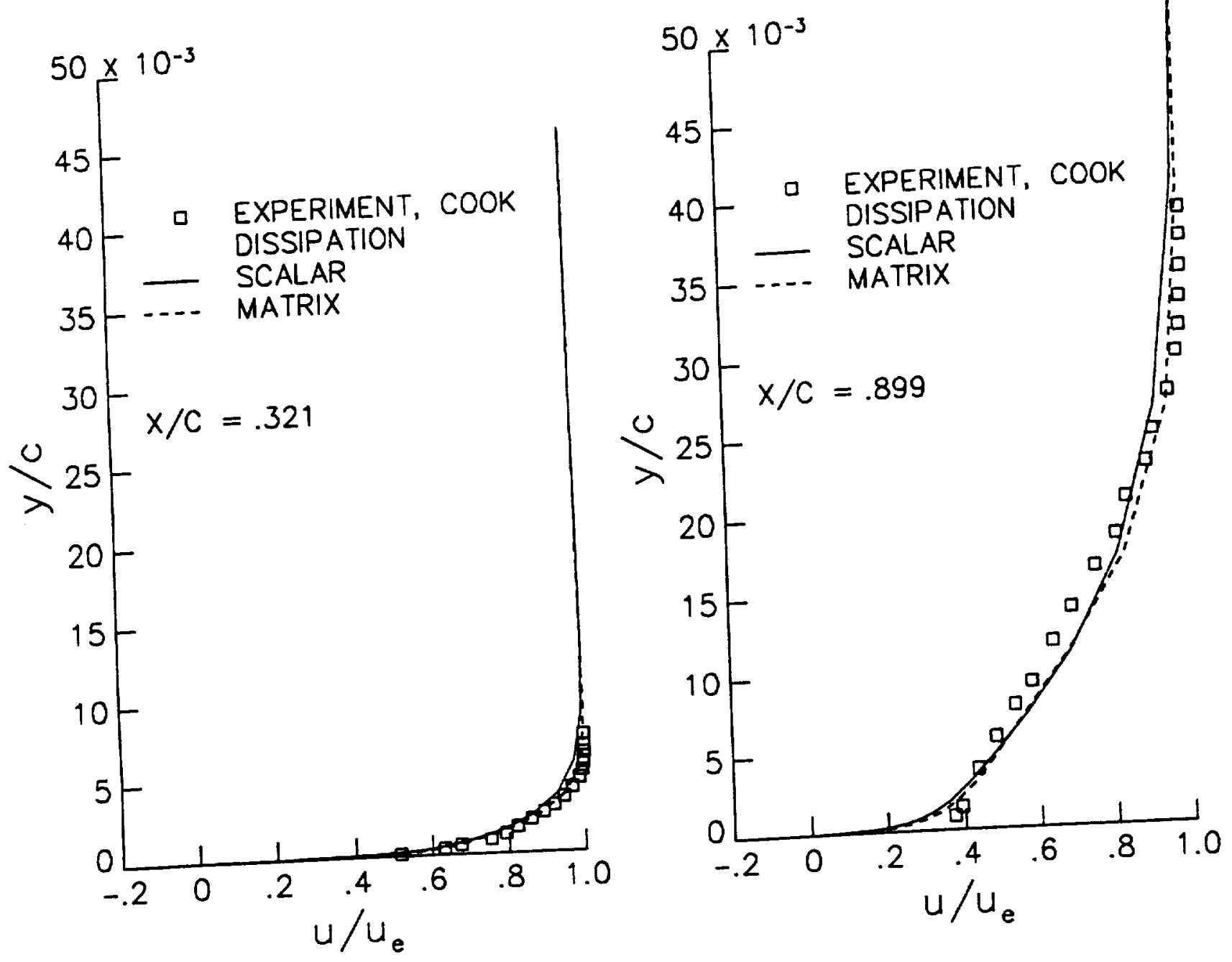

(e)

Figure 4e-4f Velocity profiles $(160 \times 32$ mesh) 

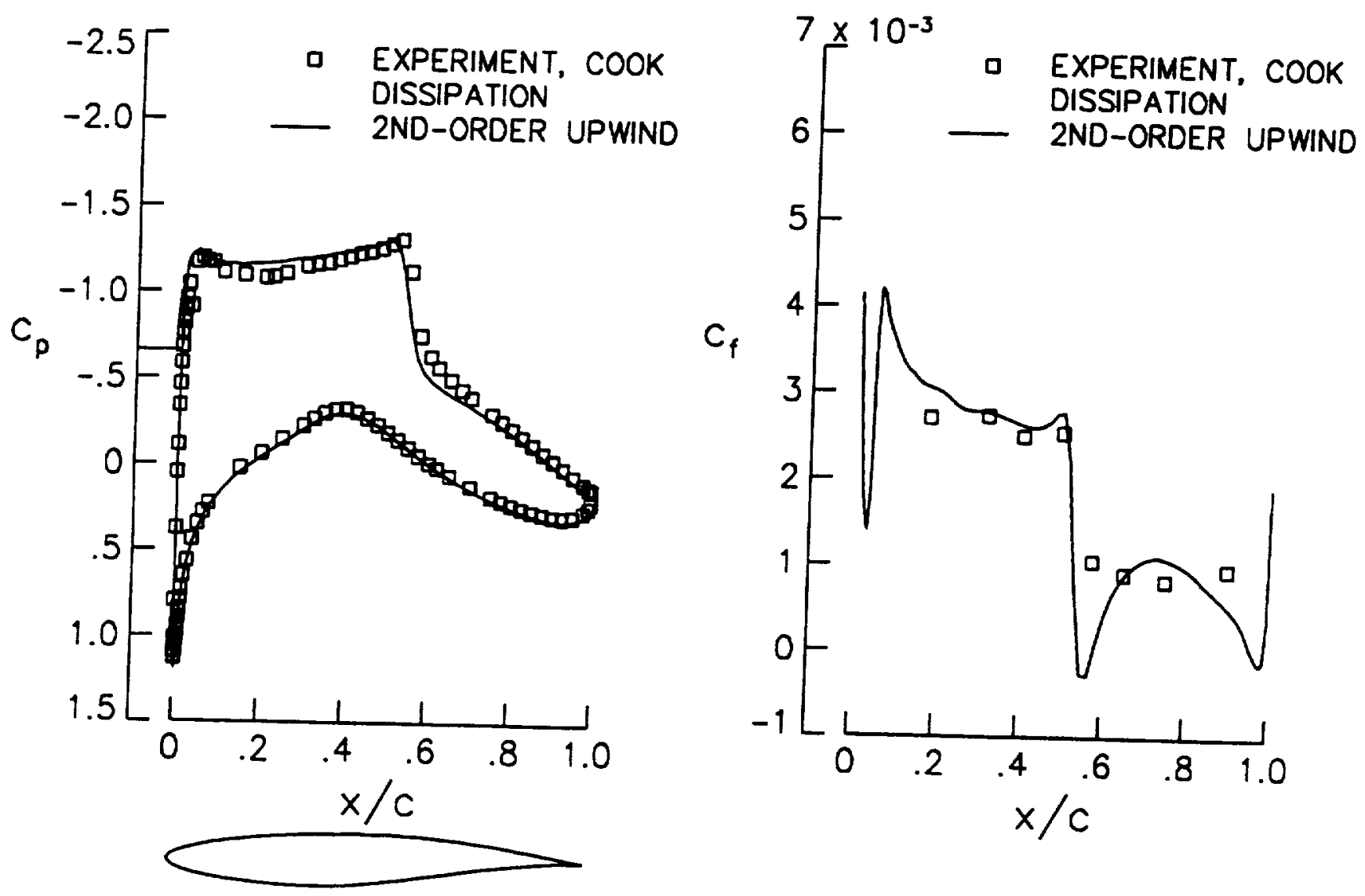

(a)

(b)

Figure 5 Second-order Roe scheme results for turbulent flow over RAE 2822 airfoil $\left(160 \times 32\right.$ mesh, $M_{\infty}=0.73, \alpha=2.79$ degrees, $\left.\operatorname{Re}_{\infty}=6.5 \times 10^{6}\right)$ :

(a) Surface pressure coefficient, (b) Upper surface skin-friction coefficient 


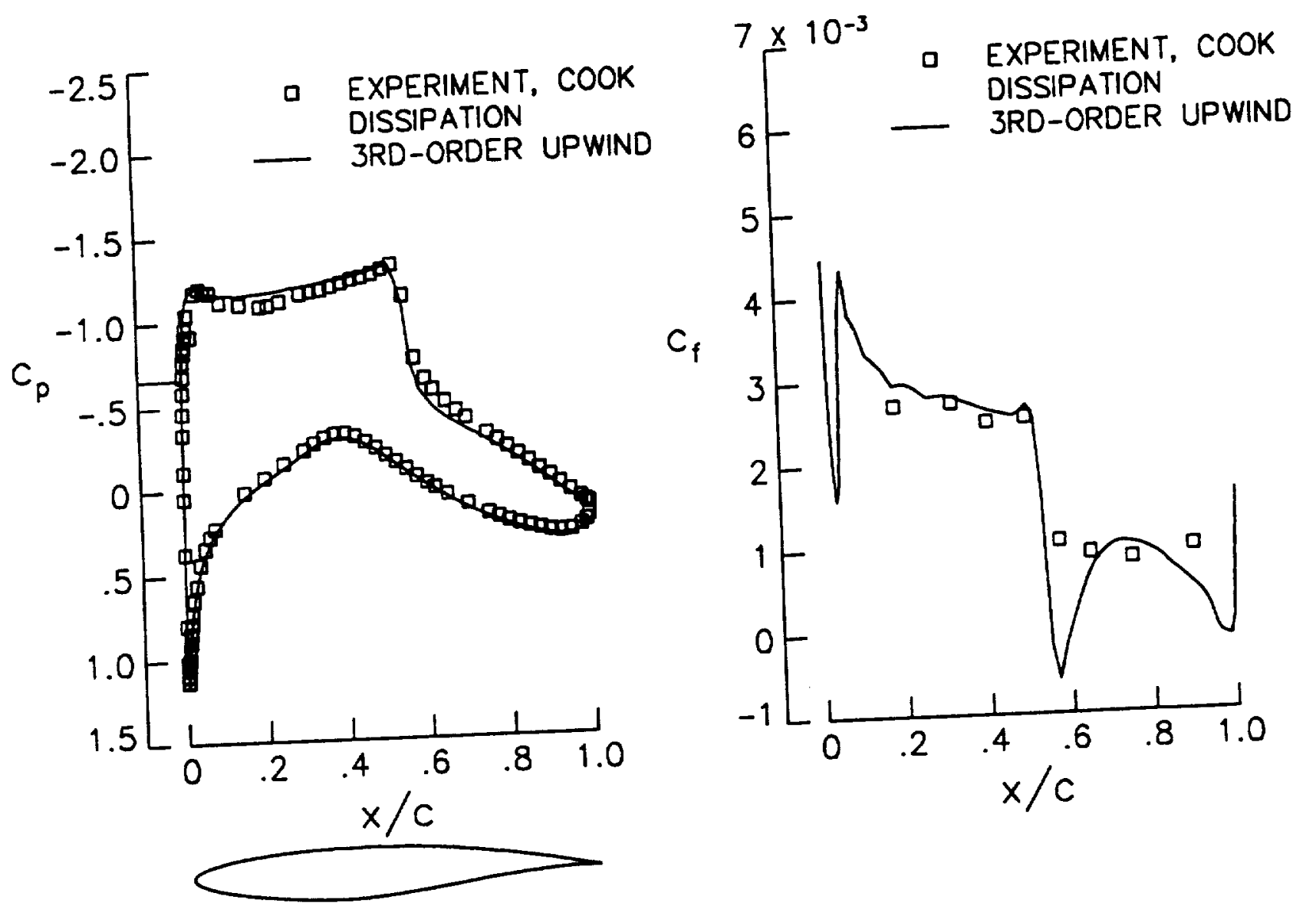

(a)

(b)

Figure 6 "Third-order" Roe scheme results for turbulent flow over RAE 2822 airfoil $\left(160 \times 32\right.$ mesh, $M_{\infty}=0.73, \alpha=2.79$ degrees, $\left.\operatorname{Re}_{\infty}=6.5 \times 10^{6}\right)$ :

(a) Surface pressure coefficient, (b) Upper surface skin-friction coefficient 

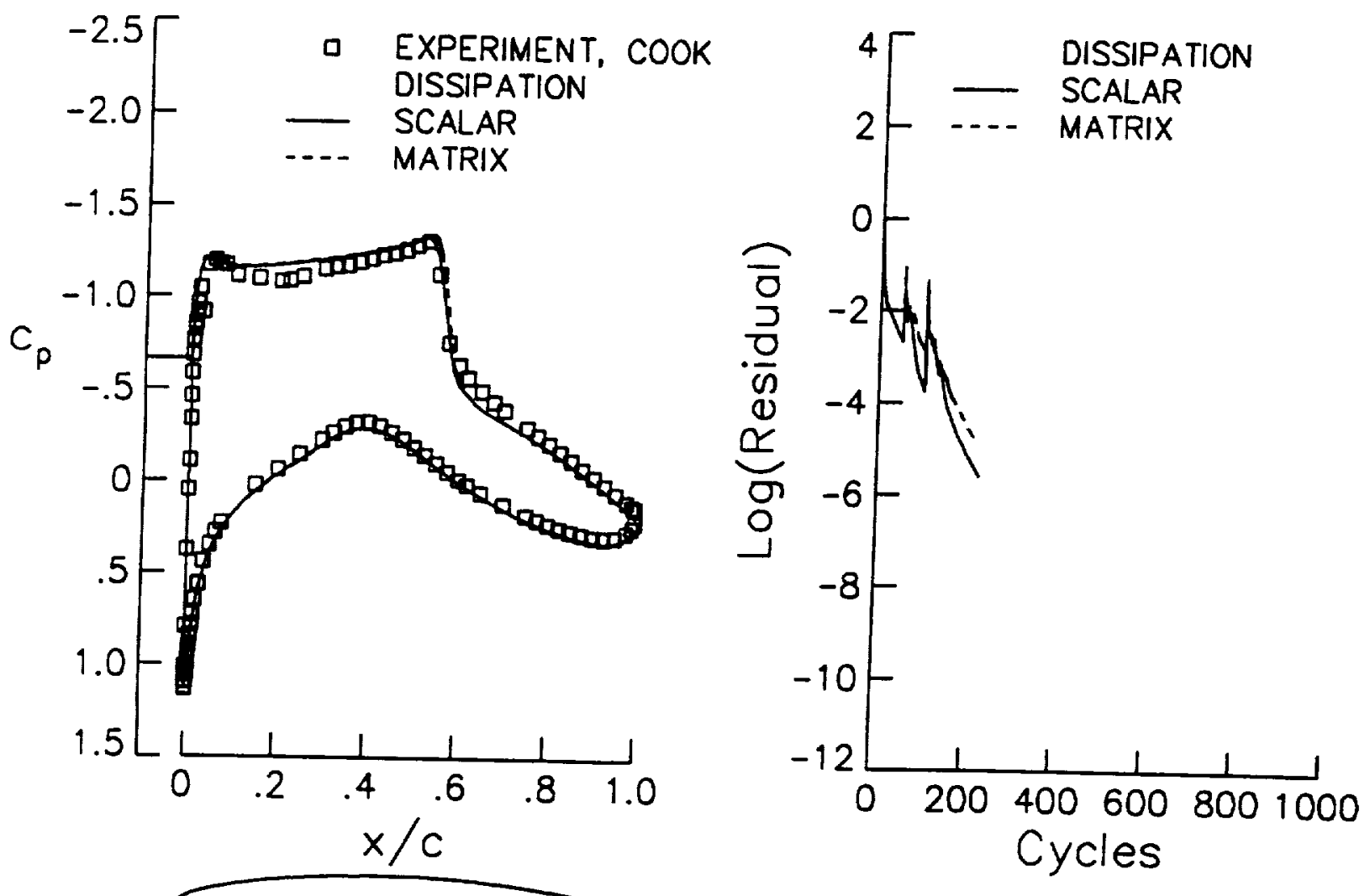

(a)

(b)

Figure 7 Central-difference scheme results for turbulent flow over RAE 2822 airfoil $\left(320 \times 64\right.$ mesh, $M_{\infty}=0.73, \alpha=2.79$ degrees, $\left.\operatorname{Re}_{\infty}=6.5 \times 10^{6}\right)$ :

(a) Surface pressure coefficient, (b) Convergence history 


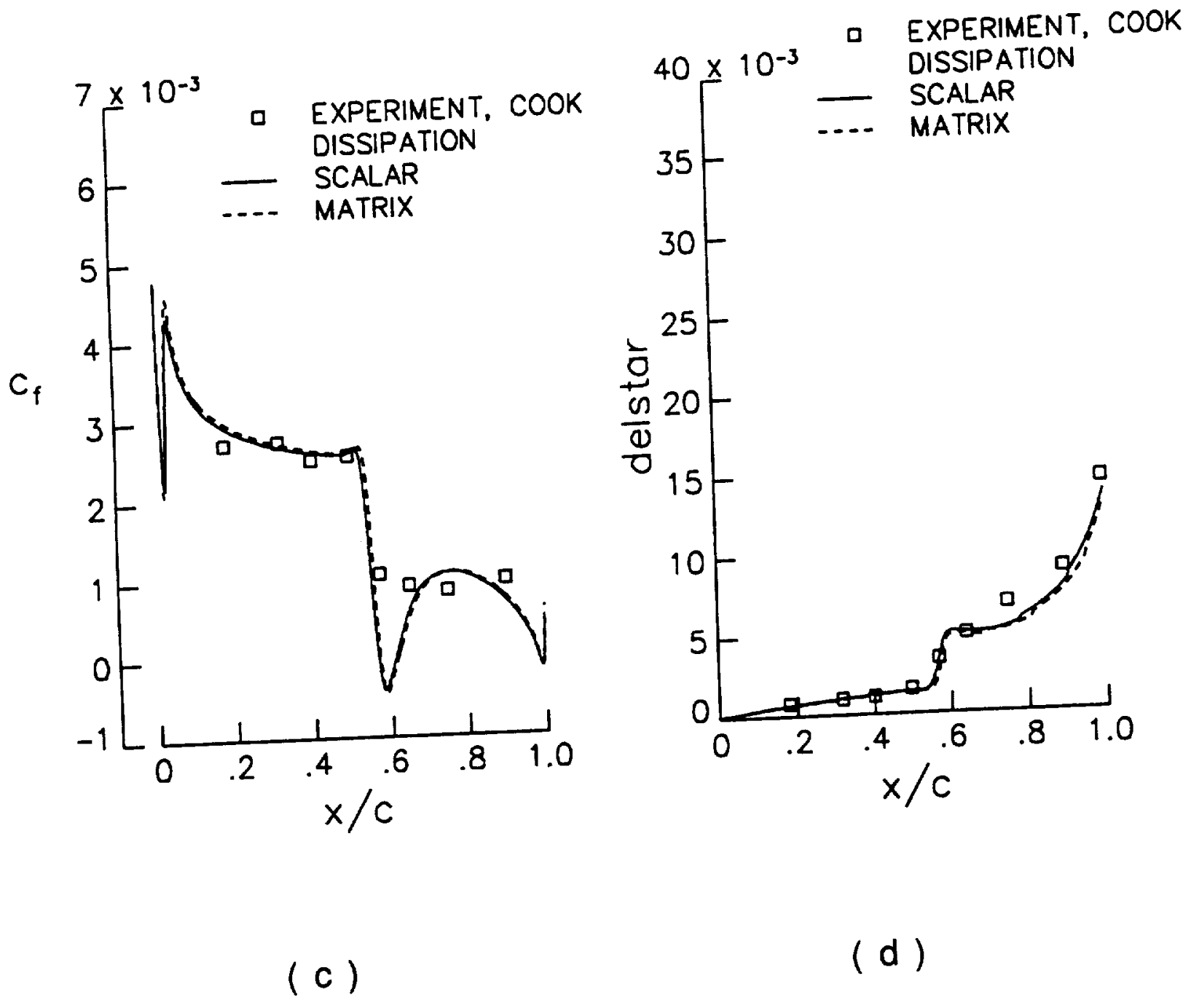

Figure 7c-7d Upper surface skin-friction coefficient $\left(c_{f}\right)$ and boundary-layer displacement thickness $(320 \times 64$ mesh $)$ 

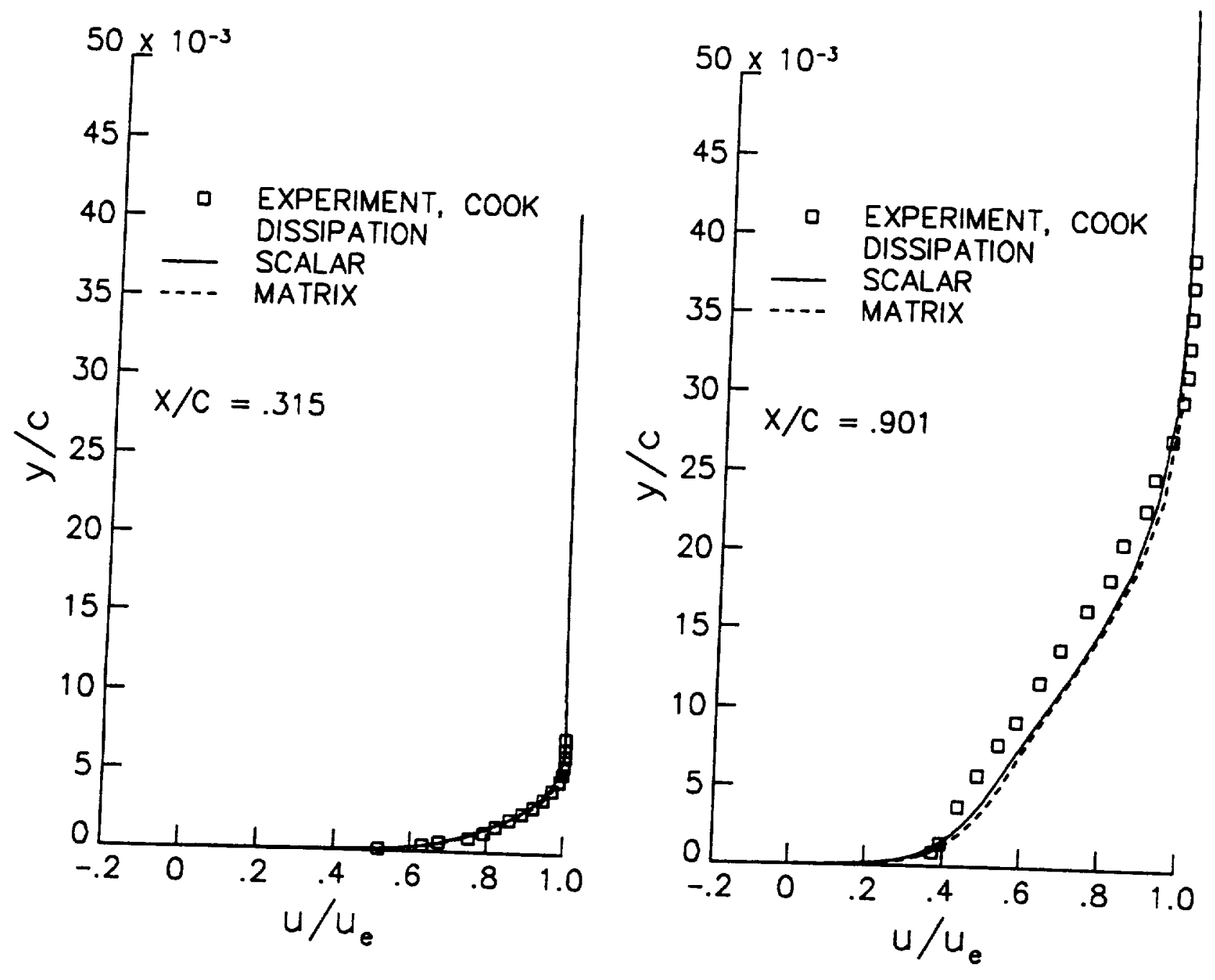

(e)

(f)

Figure $7 \mathrm{e}-7 \mathrm{f}$ Velocity profiles $(320 \times 64 \mathrm{mesh})$ 

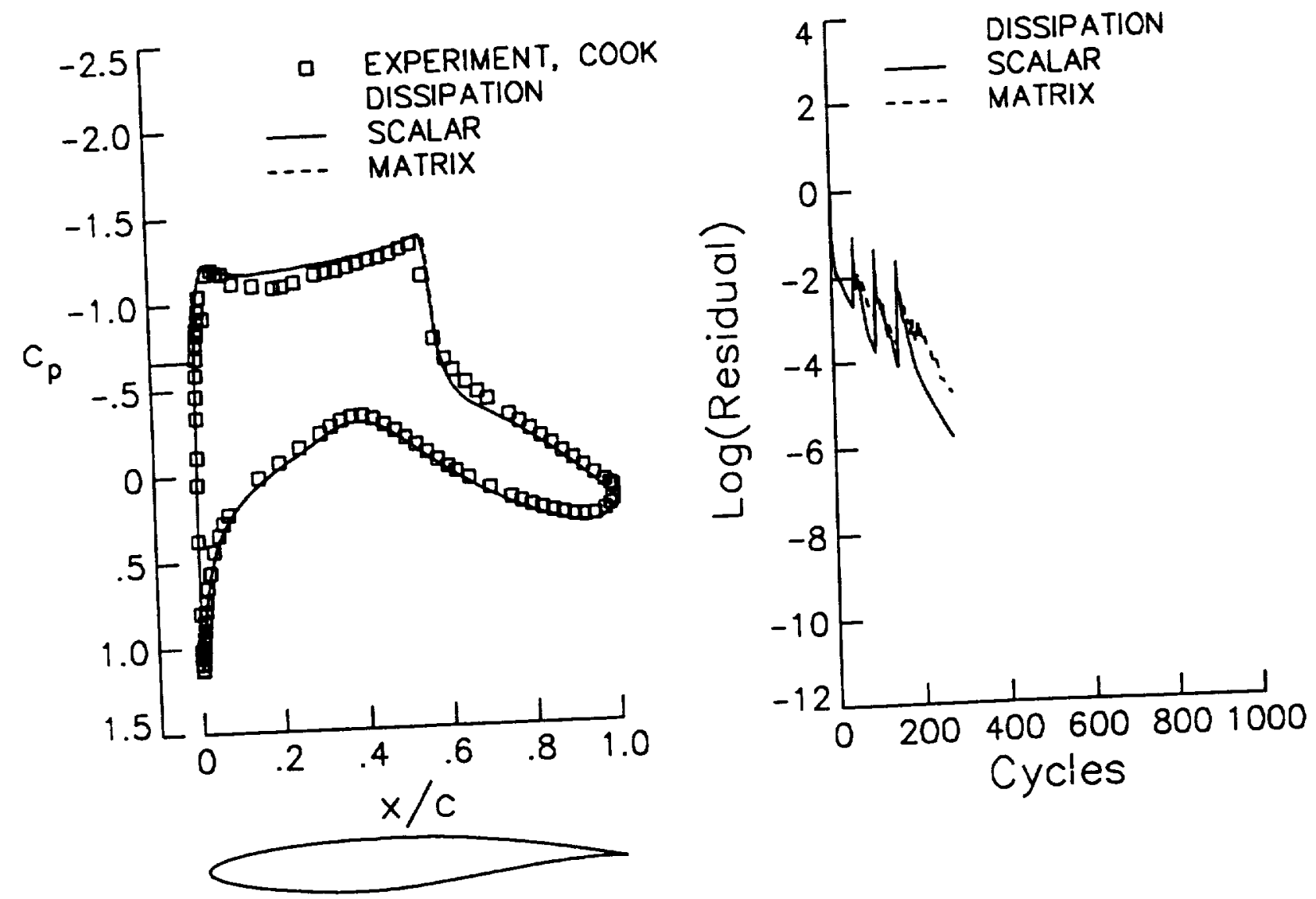

(a)

(b)

Figure 8 Central-difference scheme results for turbulent flow over RAE 2822 airfoil $\left(640 \times 128\right.$ mesh, $\mathrm{M}_{\infty}=0.73, \alpha=2.79$ degrees, $\left.\mathrm{Re}_{\infty}=6.5 \times 10^{6}\right)$ :

(a) Surface pressure coefficient, (b) Convergence history 


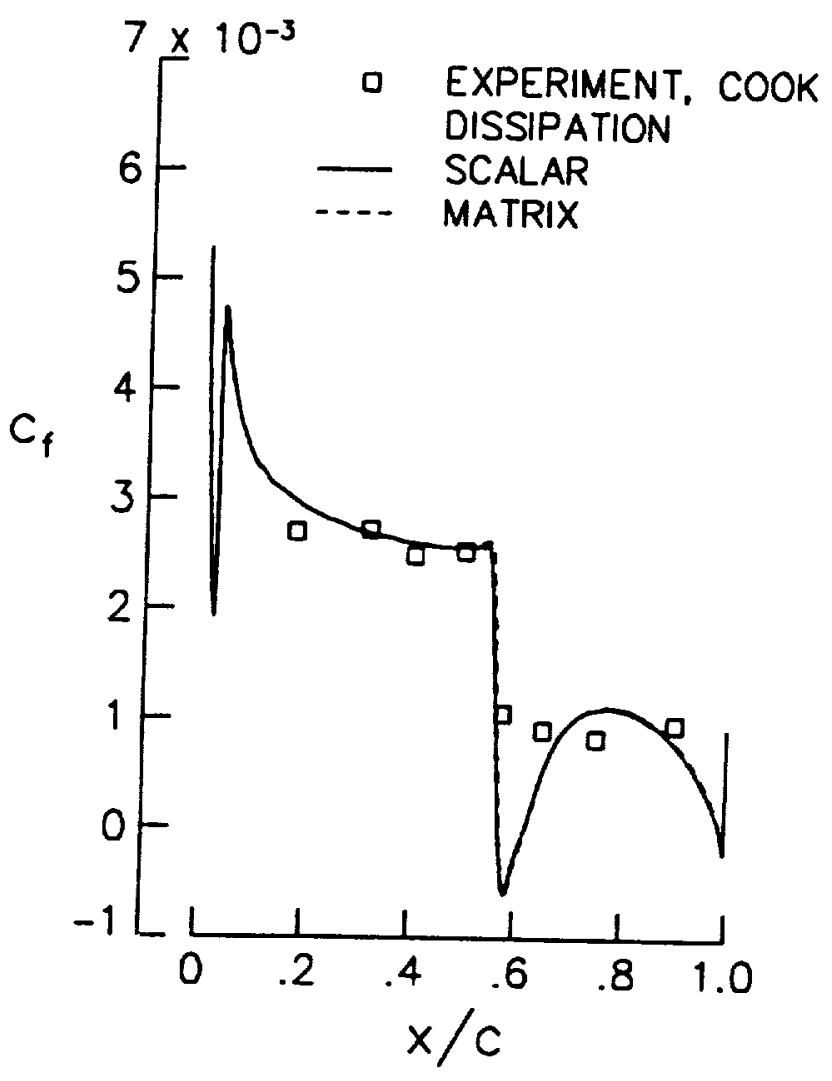

Figure 8c Upper surface skin-friction coefficient $(640 \times 128$ mesh $)$ 


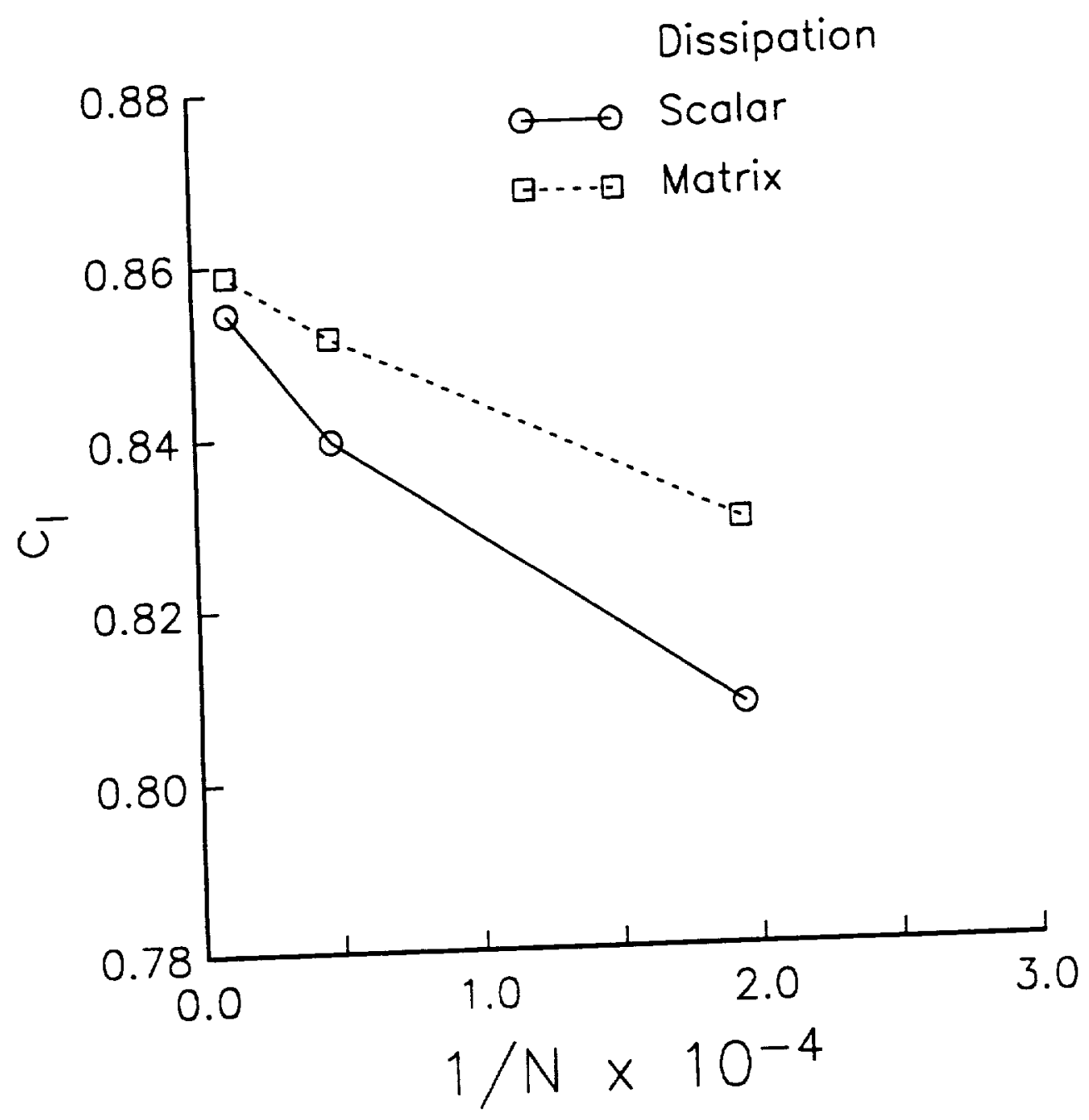

Figure 9a Variation of lift coefficient with reciprocal of number of mesh points (RAE 2822 airfoil, $\mathrm{M}_{\infty}=0.73, \alpha=2.79$ degrees, $\mathrm{Re}_{\infty}=6.5 \times 10^{6}$ ) 


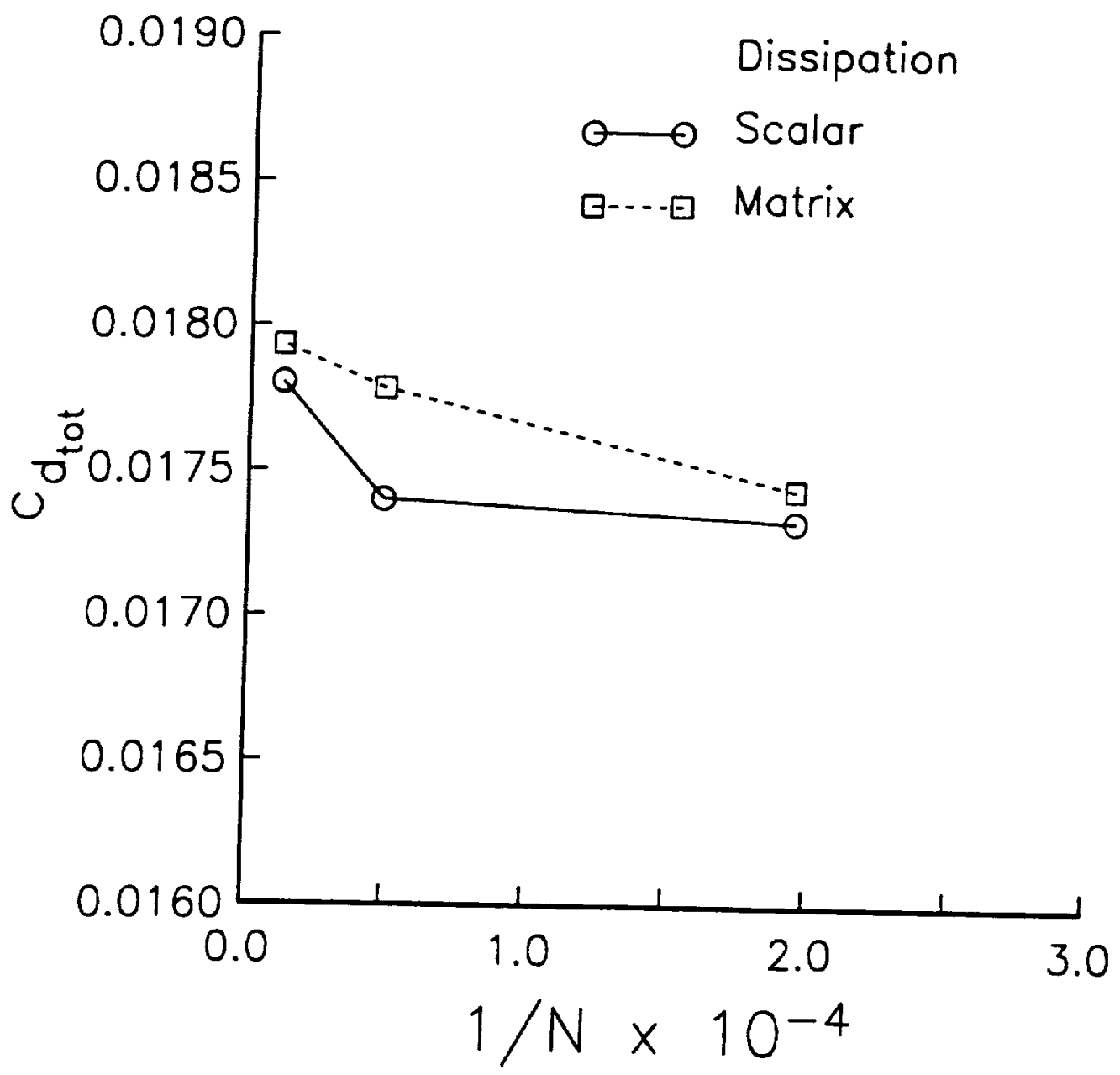

Figure 9b Variation of total drag coefficient with reciprocal of number of mesh points (RAE 2822 airfoil, $\mathrm{M}_{\infty}=0.73, \alpha=2.79$ degrees, $\operatorname{Re}_{\infty}=6.5 \times 10^{6}$ ) 


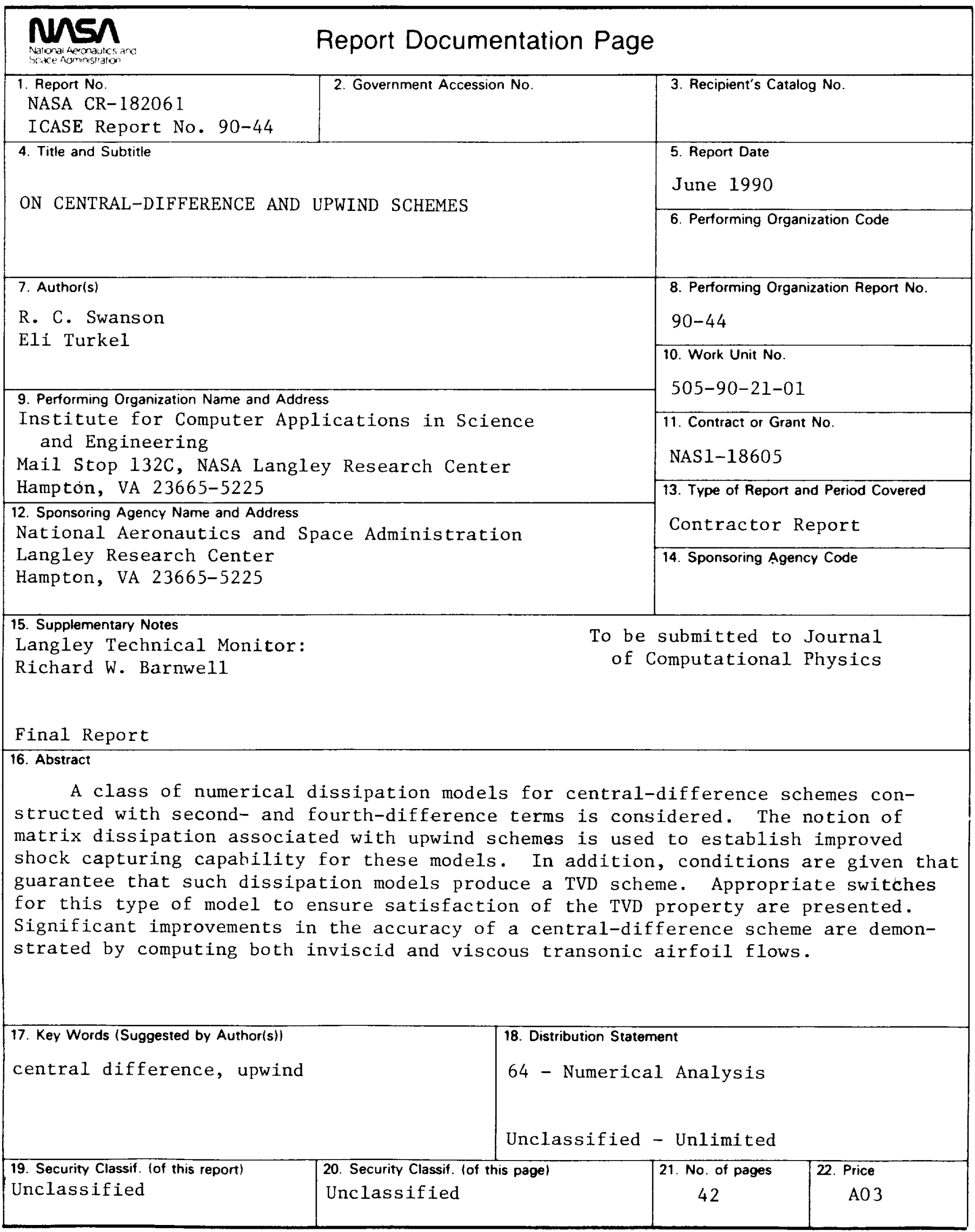


-.. 